\title{
An explanation for salinity- and SPM-induced vertical countergradient buoyancy fluxes
}

\author{
Michel A. J. de Nijs • Julie D. Pietrzak
}

Received: 18 January 2010 / Accepted: 5 January 2011 / Published online: 8 February 2011

(C) The Author(s) 2011. This article is published with open access at Springerlink.com

\begin{abstract}
Measurements of turbulent fluctuations of velocity, salinity, and suspended particulate matter (SPM) are presented. The data show persistent countergradient buoyancy fluxes. These countergradient fluxes are controlled by the ratio of vertical turbulent kinetic energy (VKE) and available potential energy (APE) terms in the buoyancy flux equation. The onset of countergradient fluxes is found to approximately coincide with larger APE than VKE. It is shown here that the ratio of VKE to APE can be written as the square of a vertical Froude number. This number signifies the onset of the dynamical significance of buoyancy in the transport of mass. That is when motions driven by buoyancy begin to actively determine the vertical turbulent transport of mass. Spectral and quadrant analyses show that the occurrence of countergradient fluxes coincides with a change in the relative importance of turbulent energetic structures and buoyancydriven motions in the transport of mass. Furthermore, these analyses show that with increasing salinity-induced Richardson number $(R i)$, countergradient contributions expand to the larger scales of motions and the relative importance of outward and inward interactions increases. At the smaller scales, at moderate $R i$, the countergradient buoyancy fluxes are physically associated with an asymmetry in transport of fluid parcels by energetic turbulent motions. At the large scales, at large $R i$, the countergradient buoyancy fluxes are physically associated with convective motions induced by buoyancy of incompletely dispersed fluid parcels which have
\end{abstract}

Responsible Editor: Alejandro Jose Souza

M. A. J. de Nijs $(\bowtie) \cdot J$. D. Pietrzak

Environmental Fluid Mechanics Section,

Delft University of Technology,

PO Box 5048, Stevinweg 1,

2600 GA Delft, the Netherlands

e-mail: m.a.j.denijs@tudelft.nl

URL: www.fluidmechanics.tudelft.nl been transported by energetic motions in the past. Moreover, these convective motions induce restratification and enhanced settling of SPM. The latter is generally the result of salinity-induced convective motions, but SPM-induced buoyancy is also found to play a role.

Keywords Countergradient buoyancy fluxes · Stratified shear flow. Available turbulent potential energy . Vertical turbulent kinetic energy · Energetic coherent structures . Convective motions

\section{Introduction}

Stratified flows occur in the atmosphere, oceans, estuaries, and lakes. They are a ubiquitous feature of environmental and geophysical flows. The turbulent transfer of momentum, heat, and mass (salinity, suspended particulate matter (SPM), contaminants) are all strongly affected by buoyancy forces. Consequently, detailed knowledge of turbulent mixing is essential to our understanding of stratified flows. Moreover, in shelf seas, estuaries, and adjacent harbor basins, salinity and SPM distributions are often related to one another (e.g., de Nijs et al. 2009, 2010b). Understanding this relationship and its consequences for the circulation and transport pathways of SPM, and for the trapping and sedimentation processes of SPM in these environments, is important. Therefore, the precise interactions between salinity and SPM-induced buoyancy on turbulent mixing are also of great interest for ecologists, estuarine, river basin, and port management.

Extensive studies on the effects of buoyancy forces on the turbulent transfer of momentum, heat, and mass have been done in the past decades. These effects have been studied using algebraic and numerical models (e.g., Munk 
and Anderson 1948; Mellor and Yamada 1974; Launder 1975; Nunes Vaz and Simpson 1994; Gerz and Schumann 1996; Burchard et al. 1998; Armenio and Sarkar 2002; Taylor et al. 2005), laboratory facilities (e.g., Itsweire et al. 1986; Rohr et al. 1988; Komori et al. 1983; Komori and Nagata 1996), and field surveys (e.g. Osborn and Cox 1972; Osborn 1980; Anwar 1983; West and Shiono 1988; West and Oduyemi 1989; Peters 1997; van der Ham 1999; Etemad Shahidi and Imberger 2006). However, these studies generally investigate the effects of buoyancy forces induced by a single active scalar on the turbulence level and associated mixing in shear flows. Here, we investigate the effects of buoyancy forces induced by two active scalars, salinity and SPM. Furthermore, we investigate countergradient transport and its role in coastal and estuarine flows.

Komori et al. (1983) were the first to identify countergradient fluxes in the outer layer of a thermally stratified open-channel flow with weak shear. In turbulent flows, the signs of the ensemble mean vertical turbulent transports of salinity and SPM are usually opposite to those of the mean gradients of salinity and SPM. These fluxes are referred to as downgradient fluxes and modeled as diffusion. However, in stratified flows, gravity oscillations can develop, which causes oscillating fluxes at a buoyancy frequency of about $1 / 2 \pi^{-1} N$ (where $N$ is the Brunt-Väisälä frequency). These oscillations become countergradient when potential energy is transferred to kinetic energy. Therefore, Gerz and Schumann (1996) introduced the term persistent countergradient flux. This term applies to countergradient fluxes when they persist for longer than half the buoyancy period. Such fluxes have been measured in thermally and salinitystratified homogeneous shear flows (Komori et al. 1983; Schumann 1987; Gerz and Schumann 1996), in unsheared stratified flows (Komori and Nagata 1996), in linearly saltstratified grid-generated turbulence (Itsweire et al. 1986; Rohr et al. 1988), in stratified flows with a gradient in turbulent properties or slightly inhomogeneous turbulent conditions (Schumann 1987; Komori and Nagata 1996), and in a tidal flow with strong SPM stratification (Dyer et al. 2004).

Schumann (1987) investigated the mechanisms driving countergradient transport in uniform stably stratified flows with second-order model equations. He concluded that this transport arises in highly, stably stratified flows when the dissipation of temperature variance is too small to balance its source. This occurs particularly for high Prandtl number flows: $P r \approx 600$ for salinity and $P r \approx 5$ for thermally stratified flows. Komori and Nagata (1996) experimentally investigated countergradient fluxes in a thermally stratified, mixing layer-type flow, with decaying turbulence in the streamwise direction. Their experiments indicated that countergradient heat transport could be explained by the relative balance between the available potential energy (APE) associated with the density variance and the vertical turbulent kinetic energy (VKE). At some point downstream, the available potential energy became larger than the vertical turbulent kinetic energy. This more or less coincided with their observations of countergradient fluxes.

Komori et al. (1983) attributed countergradient heat fluxes to intermittent buoyancy-driven turbulent motions, which become wavelike as a result of strong stratification. Based on large eddy simulation (LES) data and laboratory measurements, Gerz and Schumann (1996) proposed a conceptual model for the turbulent motions that determine the countergradient transport of momentum and heat in homogeneous stratified shear flows. They distinguished between countergradient fluxes at large and small scales. Countergradient buoyancy fluxes develop at positive $R i$ (Richardson number), first at the small scales. Pairs of overlying hairpin vortices transport fluid parcels between their legs. These energetic fluid parcels collide with each other and break up into smaller ones. After collisions have taken place, an asymmetry develops in the transport of the smaller fluid parcels. This produces a countergradient flux at the small scales. Hence, the kinetic energy of the small scales is supplied by the transfer of energy from the large energetic scales. Countergradient fluxes at large scales evolve at large $R i \quad(\approx 0.5-1)$. Physically, these motions represent parcels of fluid, remnants of transport by energetic turbulent motions in the past, which move by their own buoyancy to their equilibrium level in terms of density, and these parcels occasionally collide. Therefore, energy to the countergradient motions is supplied by conversion of available potential energy to kinetic energy.

Laboratory experiments by Komori and Nagata (1996) confirmed that countergradient heat fluxes first arose at the small scales. They also showed that with increasing stratification, they expanded to the large scales in flows with different Prandtl and Schmidt numbers. In high Prandtl-Schmidt number flows such as water, countergradient heat fluxes can occur at smaller scales of motion than found for low Prandtl-Schmidt number flows such as air. This is because in air flows, small-scale motions more rapidly lose their potential energy (due to the larger dissipation by molecular viscosity) than in water flows.

Studies on countergradient transport in the coastal and estuarine context are scarce. Earlier field studies observed a decrease in correlation coefficients between vertical turbulent transport and increasing stability. However, they attributed the decrease to internal wavelike motions (West and Shiono 1988) or to the replacement of turbulent fluctuations by internal wave fluctuations (West and Oduyemi 1989). Dyer et al. (2004) measured countergradient SPM-induced buoyancy fluxes. They ascribed 
these fluxes to the enhanced settling of SPM due to the production of inactive turbulence. They attributed this turbulence structure to the effects of internal waves on the turbulent bursting structure and the consequent interaction with the concentration profile. However, in light of the aforementioned studies, their explanations for the damping effect and countergradient buoyancy fluxes seem somewhat unsatisfactory. The decrease in correlation coefficients could be the result of a different transport mechanism by energetic turbulent structures with increasing buoyancy force instead of internal wave motions. This mechanism involves an asymmetry in transport by energetic structures induced by buoyancy, as well as buoyancy-induced transport by remnants of the energetic transports.

Advances in instrumentation have enabled researchers to study turbulence in more detail in coastal and estuarine flows. The use of high-frequency acoustic Doppler velocity meters and electric magnetic flow meters (EMFs) has become common practice. These techniques allow for the direct measurements of turbulent properties, such as kinetic energy and Reynolds (shear) stresses at a specific point in the water column (e.g., van der Ham 1999). More recently, studies by Stacey et al. (1999) and Rippeth et al. (2003) have employed the variance method (Tropea 1983; Lohrmann et al. 1990) to determine Reynolds shear stresses with acoustic Doppler current profilers (ADCPs) over the whole water column. However, this measurement technique does not allow for direct measurements of the turbulent buoyancy flux. Other studies (e.g., Peters 1997; Etemad Shahidi and Imberger 2006) estimate buoyancy fluxes from microprofiler data using the methods by Osborn and Cox (1972) and Osborn (1980). Studies where buoyancy fluxes have been directly measured are scarce.

While it has been indicated that buoyancy fluxes can become countergradient, little direct observational evidence in the estuarine and coastal context can be found in the literature. There is neither a satisfactory explanation for countergradient buoyancy fluxes, nor an explanation for the precise effects of the interactions between salinity- and SPM-induced buoyancy on turbulent mixing and the associated turbulent transport of mass. Here, we present high-frequency measurements, collected with an anchored station, in a low energetic stratified environment. The turbulent flow structure is examined from velocity profiles derived from ADCP data and from direct measurements of Reynolds stresses and buoyancy fluxes with EMFs, conductivity-temperature-depth, and turbidity (MEX) sensors. This setup allows us to study the behavior of turbulent kinetic energy, Reynolds (shear) stresses, and salinity- and SPM-induced buoyancy fluxes in the region near the bed, over a period of about a day, on April 14, 2005 and on April 16, 2006.
In Section 2, the measuring setup, the statistical treatment of data, quadrant analysis, and spectral analysis are described. In the following section, a mathematical explanation of the countergradient buoyancy fluxes is presented using transport equations for the buoyancy flux and density variance. Here, the relative balance between $\mathrm{APE}$ and VKE in the balance equation for the buoyancy flux is investigated. We introduce a discriminator parameter based on the vertical Froude number to determine the onset of countergradient transport. Here, we explore an alternative stratification discriminator parameter to the traditionally used $R i$ and Richardson flux $\left(R i_{\mathrm{f}}\right)$ numbers. In Section 4 , the variability of the tidal current structure, salinity, SPM, turbulent kinetic energy, and shear stress is characterized, and then the observations of countergradient fluxes are presented. Subsequently, the development of the discriminator parameter is compared with the occurrence of salinity- and SPM-induced countergradient buoyancy fluxes. The scales and types of motions are examined through spectral and quadrant analyses. In Section 5, the results are compared with the conceptual model first proposed by Gerz and Schumann (1996). Then, we extend their theory to inhomogeneous turbulence. The dominant contribution of salinity-induced buoyancy to the density variance indicates that settling of SPM is generally enhanced by salinity-induced convective motions. Consequently, we have also identified a new sedimentation mechanism. This mechanism contributes to the trapping efficiency of SPM in harbor basins at the fresh-saltwater interface.

\section{Methods}

\subsection{Location}

The lower river branches of the rivers Rhine and Meuse form the so-called Rhine-Meuse Estuary (Fig. 1). The upestuary borders, those no longer affected by tides, are approximately located at the cities of Hagestein, Tiel, and Lith (outside the domain of Fig. 1), along the rivers Lek, Waal, and Meuse, respectively. The down-estuary borders are at Hook of Holland and at the Haringvliet sluices, which form open and regulated connections to the North Sea. The Port of Rotterdam and Botlek Harbor (Fig. 1) are part of this estuarine system. A measuring rig was deployed in the turning circle of the Botlek Harbor basin (Fig. 1). The rig was located at $80,932^{\circ} \mathrm{E}, 433,861^{\circ} \mathrm{N}$, about $50 \mathrm{~m}$ from the edge of the quay. This distance is about three to four times the local water depth, which is considered sufficient to exclude possible effects of the quay wall on the measurements. The rig was positioned in front of a shortterm mooring facility for inland shipping (pontoon) to avoid interference with seagoing ship traffic. 
Fig. 1 Rhine-Meuse estuary (above), Port of Rotterdam (below), and Botlek Harbor (upper right corner). The location of the rig is shown in the latter panel

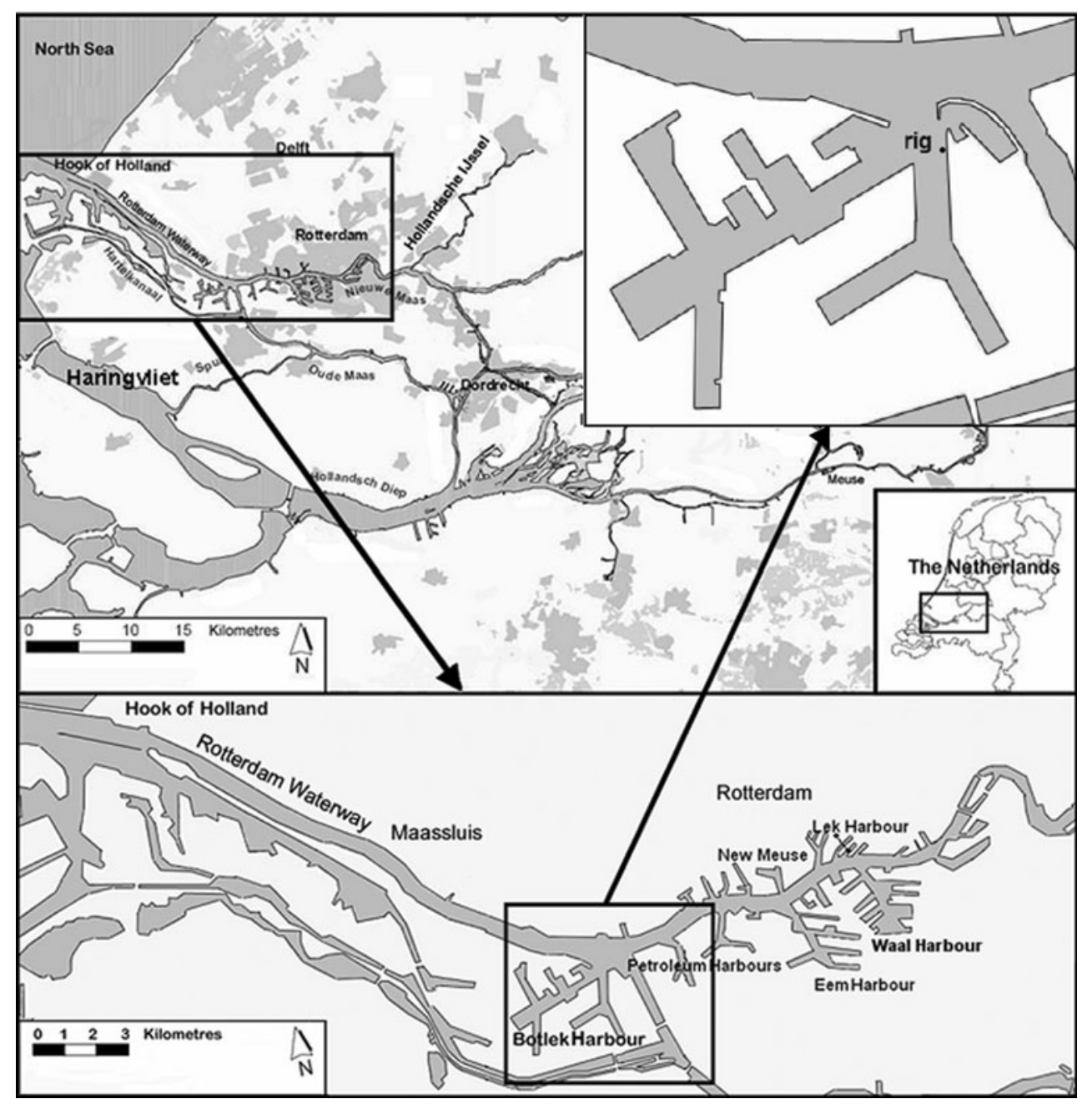

\subsection{Setup}

Six EMF flow meters, three MEX-type turbidity sensors, and three conductivity and temperature sensors were mounted on the rig and a pole attached to the rig. Three combinations of EMF, conductivity, and MEX "measuring units" were mounted at respectively $0.30,0.70$, and $3.20 \mathrm{~m}$ above the bed (mab; see Fig. 2). The 3D flow field was measured by two EMFs with discus-type sensing heads. These EMFs have an accuracy of about $1.5 \%$ of measured value. The offsets were measured in situ, prior and after deployments. The distance between two different sensors at the same level was approximately $0.05 \mathrm{~m}$. This distance has been chosen so that the magnetic fields of the EMFs do not interfere with each other. A Campbell Scientific CR5000 data logger recorded the instrument signals with $10 \mathrm{~Hz}$. High-frequency measuring $(10 \mathrm{~Hz})$ conductivity sensors with small measuring volumes were combined with temperature sensors with a measuring frequency of $0.3 \mathrm{~Hz}$. These sensors have an accuracy of about $0.01 \mathrm{mS}$ $(\mathrm{cm})^{-1}$ and $0.01{ }^{\circ} \mathrm{C}$, respectively. Two RD instruments, Inc. $1,200-\mathrm{kHz}$ Workhorse ADCPs were mounted on the rig. The angle of each beam with the vertical is $20^{\circ}$. The downward-looking ADCP operated in mode 5 with $0.05-\mathrm{m}$ depth cell sizes and a measuring frequency of $6 \mathrm{~Hz}$. The upward-looking ADCP operated in mode 12 with $0.20-\mathrm{m}$ depth cell sizes. An ensemble was determined over five sub-pings. The frequency response was set to $2 \mathrm{~Hz}$. The profiling range was chosen short enough to prevent successive pings interfering with each other. The uncertainty associated with the along-beam velocity estimates for the upward- and downward-looking ADCPs are 0.0228 and $0.006 \mathrm{~ms}^{-1}$, respectively (RDI Plan Software). The axes of the ADCPs and EMFs were aligned with the predominant flow direction approximately directed along the quay.

Main flow results from April 14, 2005 are presented in Fig. $3 \mathrm{~b}$. The band within -10 and $-8 \mathrm{~m}$ (Fig. $3 \mathrm{~b}$ ) is due to the blanking distances of the upward- and downwardlooking ADCPs. These distances amount to 1.8 and $0.50 \mathrm{~m}$, 
Fig. 2 Measuring rig (AMF) equipped with ADCPs and a measuring pole with additional instruments
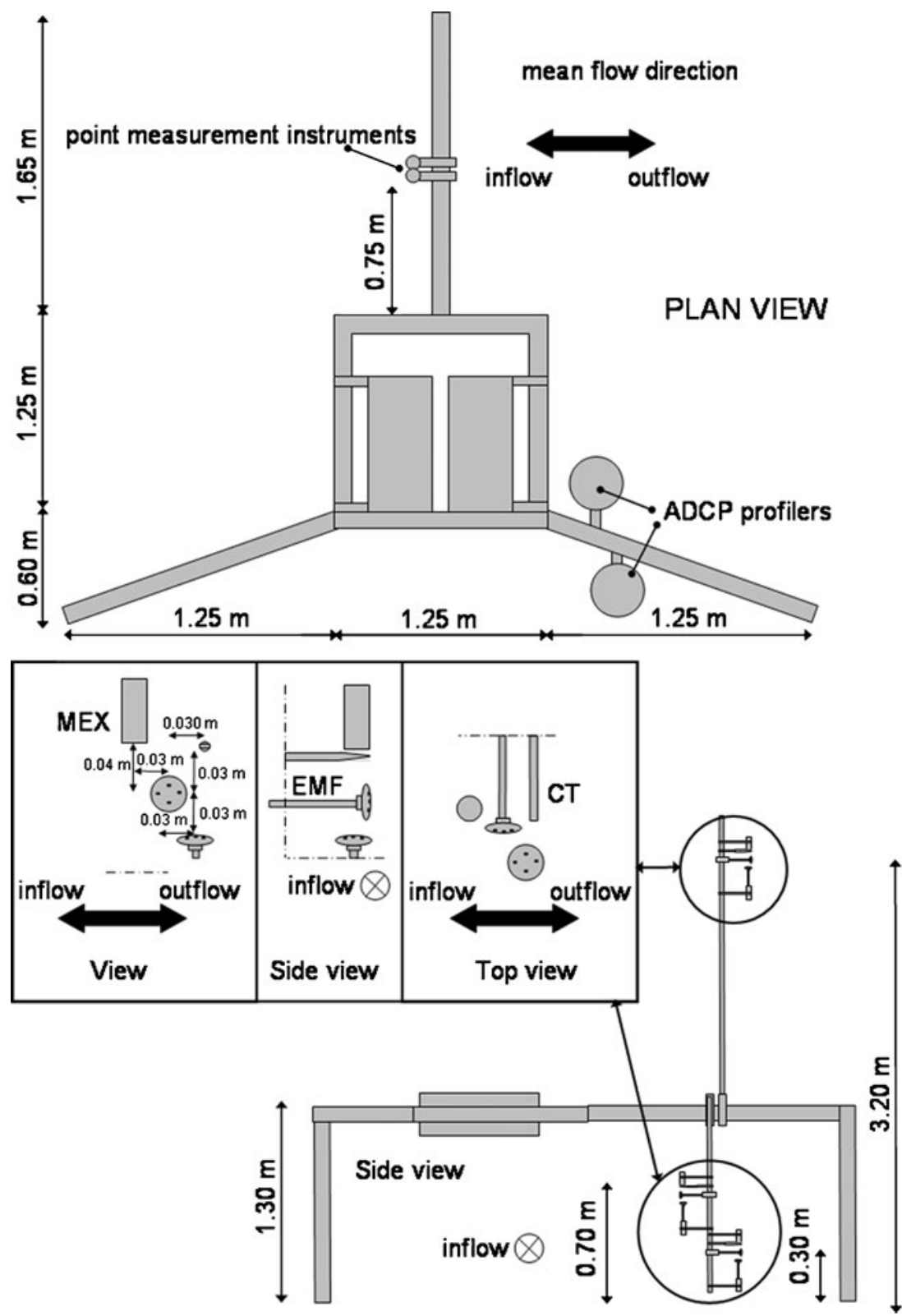

respectively. Therefore, a part of the water column is not measured. The interface between opposite velocities is located at about -9 to $-8 \mathrm{~m}$ (see also de Nijs et al. 2009), which is about $0.8-1.8 \mathrm{~m}$ above the top of the tubular frame. Therefore, we expect that there are no potential interactions with the rig. Moreover, we inspected the energy density spectra of velocity, salinity, and SPM fluctuations and found no evidence of vortex shedding by the rig. We evaluated this by inspecting the energy density at a frequency range around the so-called vortex shedding frequency, defined as $f=S t\langle U\rangle(D)^{-1}$, with $S t$ the shedding Strouhal number taken equal to 0.2 (Zdravkovich $1997),<U>$ the primary ensemble mean velocity component, and $D$ the diameter of the tubular frame.

\subsection{Data processing and standard statistical analysis}

Stationarity tests were performed to select an appropriate averaging period to determine the ensemble mean flow and turbulence properties (e.g., Bendat and Piersol 1971 in Soulsby 1980; West et al. 1986). The data were averaged using an unweighted moving average window of $10 \mathrm{~min}$. A sensitivity study concerning spike identification and removal found only a minor quantitative effect on the turbulence statistics. Velocity signals were corrected for tilt. Salinity was calculated from conductivity and temperature measurements using the 1978 practical salinity scale equations (IEEE 1980). The practical salinity scale 1978 defines salinity in terms of a conductivity ratio. We apply 
Fig. 3 Data recorded at the anchored station during the survey on April 14, 2005. a Graph shows, from top to bottom, primary velocity component, salinity, SPM concentration, turbulence kinetic energy, and vertical turbulent transport of horizontal momentum, respectively. Time (hours) is shown on the $x$-axis. Data at $0.30 \mathrm{~m}$ (dashed thick line) and $3.20 \mathrm{~m}$ (thin line) from the bed are shown. b Graph showing the vertical structure of the primary velocity component (positive $=$ outflow). The time of the survey is shown on the $x$-axis and position within the water column on the $y$-axis. In the lower part of the graph, salinity (above) and SPM concentration (below) are also shown as two time series against a secondary $y$-axis on the left and right, respectively. The water level and harbor bed are shown by the thin black lines
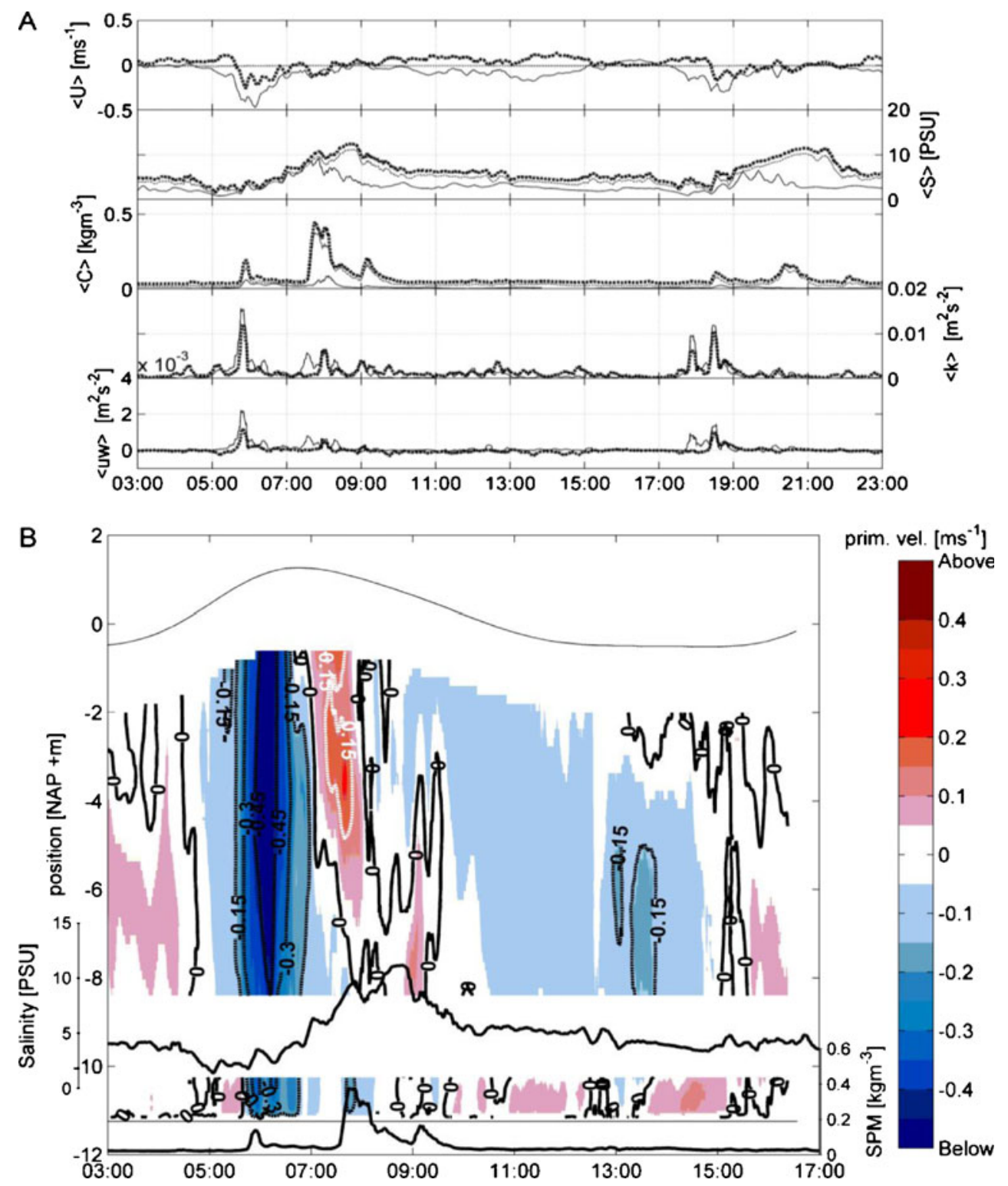

the commonly used practical salinity unit (PSU) as the physical unit for salinity.

\subsection{Analysis of the types of motions through quadrant and spectral analysis}

In the quadrant analysis (e.g., Lu and Willmarth 1973), turbulent motions (ejections, sweeps, and outward and inward interactions) are classified according to the sign of horizontal primary velocity fluctuations $(u)$ and vertical velocity fluctuations $(w)$ and SPM concentration fluctuations $(c)$. The notation used is as follows: The instantaneous value of the primary horizontal velocity component is written as the sum $U=\langle U\rangle+u$ of the ensemble averaged velocity $\langle U\rangle$ and the fluctuating velocity $u$. Similar notations are used for the vertical velocity component $(W)$, salinity $(S)$, and SPM $(C)$. The quadrants in Table 1 and Fig. 12 depict negative main flow $(<U><0)$ : outward interactions (Q1: $u<0, c<0, w>0)$, ejections (Q2: $u>0, c>$ $0, w>0)$, inward interactions (Q3: $u>0, c>0, w<0)$, and sweeps (Q4: $u<0, c<0, w<0$ ). The contribution of the individual quadrants to the vertical turbulent transport of momentum $(<u w>)$, salinity $(<s w>)$, and SPM $(<c w>)$ are expressed by the fractional time $\left(T_{i}\right)$ and contribution $\left(R S_{i}\right)$.

$$
\begin{aligned}
& R S_{i}=\frac{1}{\langle c w\rangle} \frac{1}{L} \int_{t=0}^{L} c \cdot w \cdot I_{i}(t) \mathrm{d} t \\
& T_{i}=\frac{1}{L} \int_{t=0}^{L} I_{i}(t) \mathrm{d} t
\end{aligned}
$$

in which $i$ varies from 1 to $4, t$ is time, $I$ is the detection function with $I_{i}(t)=1$ for $|c \cdot w|>0$ in the $i$ th quadrantotherwise $I_{i}(t)=0$ - and $L$ is the record length. The results of the quadrant analysis are shown in Table 1. Only the results 
Table 1 Results of the quadrant analysis

\begin{tabular}{|l|c|c|c|c|c|c|c|c|}
\hline \multirow{2}{*}{ Time } & \multicolumn{2}{|l}{$<\mathrm{uw}>$} \\
\cline { 2 - 9 } & T1 & T2 & T3 & T4 & T1 & T2 & T3 & T4 \\
& RS1 & RS2 & RS3 & RS4 & RS1 & RS2 & RS3 & RS4 \\
\hline $05: 50 \mathrm{~h}$ & $16 \%$ & $34 \%$ & $22 \%$ & $28 \%$ & $21 \%$ & $28 \%$ & $20 \%$ & $31 \%$ \\
$0.30 \mathrm{mab}$ & -0.41 & 0.64 & -0.31 & $1.06 \Downarrow$ & -0.59 & $1.26 \Uparrow$ & -0.47 & 0.78 \\
\hline $07: 40 \mathrm{~h}$ & $24 \%$ & $26 \%$ & $23 \%$ & $27 \%$ & $28 \%$ & $22 \%$ & $24 \%$ & $25 \%$ \\
$0.30 \mathrm{mab}$ & -1.79 & $2.38 \Uparrow$ & -1.70 & 2.09 & 2 & -1.72 & 2.15 & $-1.44 \Downarrow$ \\
\hline $07: 48 \mathrm{~h}$ & $21 \%$ & $30 \%$ & $21 \%$ & $28 \%$ & $30 \%$ & $20 \%$ & $28 \%$ & $22 \%$ \\
$3.20 \mathrm{mab}$ & -0.70 & 0.74 & -0.47 & $1.32 \Downarrow$ & 0.52 & -0.25 & 0.90 & $-0.19 \Downarrow$ \\
\hline
\end{tabular}

The records at 0740 hours are also shown in Fig. 12. The dominance of upward or downward motions is indicated by arrows and thick line borders around the table cells. Around 0550 hours, $R i$ values near the bed are below the threshold value of 0.25 , and both momentum and buoyancy fluxes are downgradient. Around 0740 hours and 0748 hours, $R i$ values are above the threshold value of 0.25 . At 0740 hours, SPMinduced buoyancy and at 0748 hours salinity-induced buoyancy flux determine the buoyancy flux balance. Note that around 0740 hours, the vertical turbulent transport of salinity at 0.30 mab does not show (Fig. 4, third panel) any substantial variation and that its value remains close to zero

of the quadrant analysis for the event at 0740 hours at $0.30 \mathrm{~m}$ above bed are presented (Fig. 12). The quantities $T_{i}$ and $R S_{i}$ are shown as labels in each quadrant of the graphs.

Nezu and Nakagawa (1993, p. 184) report that sweep events dominate the fractional time, and ejections the fractional distribution of $\langle u w\rangle$, for equilibrium turbulence over smooth beds. Nezu and Nakagawa (1993) also show field data which indicate that the dominant contribution by either sweep or ejection events to the fractional contribution of $\langle u w\rangle$ varies with height above the bed. Field measurements by West and Oduyemi (1989) and Kawanisi and Yokosi (1993) indicate that during certain tidal phases, the contribution of ejections prevails over sweeps and vice versa. An explanation for this different behavior was not given in these studies. Therefore, the global distribution over the individual quadrants is considered important here. For further discussion, see Section 4.5.

The scales of motions contributing to (countergradient) buoyancy fluxes can be deduced by examining the phase angle and co-spectral density of the $\langle u w\rangle,\langle s w\rangle$, and $\langle c w\rangle$ co-spectra $\left(C_{\mathrm{o}}\right)$. The phase angle can be defined by

$\tan (\Delta \varphi)=\frac{Q_{\mathrm{u} \rho w}}{C_{\mathrm{o} \rho w}}$

where $\Delta \varphi$ is the phase angle, $Q_{\mathrm{u}}$ is the quadrature spectrum and $C_{\mathrm{o}}$ is the co-spectrum (shown in Figs. 13, 14, 19 in Appendix 3 and Figs. 20 and 21 in Appendix 4). When $0 \leq 1$ $\Delta \varphi \mid<1 / 2 \pi, \quad\langle s w\rangle$ and $\langle c w\rangle$ are positive. This indicates mixing of density by energetic turbulent structures. When $1 / 2 \pi<|\Delta \varphi| \leq \pi,\langle s w>$ and $\langle c w>$ are negative. This indicates that energetic turbulent structures are suppressed and buoyancy-driven motions, associated with the rising of lighter and sinking of heavier fluid parcels, determine the turbulent structure. Internal waves are typically associated with $|\Delta \varphi| \approx 1 / 2 \pi$ (e.g., West and Shiono 1988; West and Oduyemi 1989). Further discussion is given in Section 4.5.

\section{Interpretation of countergradient fluxes using flux and variance budgets}

The balance equations, for the buoyancy fluxes and density variance, are examined in order to explain the evolution of the buoyancy flux with stratification. These equations can be derived from the Navier-Stokes and Reynolds averaged Navier-Stokes equations of momentum, continuity, and scalar transport (e.g., Mellor and Yamada 1974; Launder 1975). The equations have been simplified through the use of the boundary layer approximation which amounts to neglecting the horizontal advection and diffusion terms in the transport equations

$\frac{\partial\langle\rho w\rangle}{\partial t}=-\left\langle w^{2}\right\rangle \frac{\partial\langle\rho\rangle}{\partial z}\left(1-\frac{\mathrm{APE}}{\mathrm{VKE}}\right)+\phi_{\langle\rho w\rangle}-\varepsilon_{\langle\rho w\rangle}$

$\frac{\partial\left\langle\rho^{2}\right\rangle}{\partial t}=-2\langle\rho w\rangle \frac{\partial\langle\rho\rangle}{\partial z}-\varepsilon_{\left\langle\rho^{2}\right\rangle}$

in which $\emptyset_{<\rho w\rangle}$ depicts the redistribution of $\langle\rho w\rangle$ by pressure fluctuations; $\varepsilon_{<\rho w>}$ and $\varepsilon_{<\rho^{2}}$ are the dissipation rates of $\langle\rho w\rangle$ and $\left\langle\rho^{2}\right\rangle$, respectively. $\rho=\rho_{0}+\gamma_{\mathrm{c}} \cdot C+\gamma_{\mathrm{s}} \cdot S$, where $\rho_{0}$ is a reference density and $\gamma$ an expression to convert salinity $\left(\gamma_{\mathrm{s}} \approx 0.72 \mathrm{~kg}(\mathrm{~m})^{-3}(\mathrm{PSU})^{-1}\right)$ and SPM concentration $\left(\gamma_{\mathrm{c}}=\left(\rho_{\mathrm{s}}-\rho_{\mathrm{w}}\right)\left(\rho_{\mathrm{w}}\right)^{-1} \approx 0.62\right.$, where $\rho_{\mathrm{w}}$ and $\rho_{\mathrm{s}}$ are the densities of water and sand particles, respectively) to density (e.g., West and Oduyemi 1989), as indicated by the subscripts $\mathrm{s}$ and $\mathrm{c}$, respectively. 
The behavior of the vertical salinity- and SPM-induced buoyancy fluxes $\left(\left\langle\gamma_{s} s w\right\rangle,\left\langle\gamma_{c} c w\right\rangle\right)$ may be examined using only the density variance balance (Eq. 4 ) and the substitution of $\rho=\rho_{0}+\gamma_{\mathrm{s}} \cdot S$ and $\rho=\rho_{0}+\gamma_{\mathrm{c}} \cdot C$, respectively. The buoyancy flux $(<\rho w\rangle)$ may become countergradient when $1 / 2 \cdot \partial<\rho^{2}>\cdot(\partial t)^{-1}+\varepsilon_{<\rho^{2}>}<0$. However, a distinct correlation between $\left\langle\gamma_{s} s w\right\rangle,\left\langle\gamma_{c} c w\right\rangle$, and $1 / 2 \cdot \partial\left\langle\left(\gamma_{s} s\right)^{2}\right\rangle$ $(\partial t)^{-1}, 1 / 2 \cdot \partial<\left(\gamma_{c} c\right)^{2}>(\partial t)^{-1}$, respectively, could not be discerned from the measurements collected on April 14, 2005 and April 16, 2006 at 0.30 mab. Komori and Nagata (1996), based on work by Schumann (1987), used the ratio of APE to VKE in Eq. 3 to explain the occurrence of countergradient transport in slightly non-uniform stratified shear flow with decaying turbulence in the streamwise direction. It is shown here that the ratio of VKE to APE can be interpreted as the square of a vertical Froude number $\left(F r_{\mathrm{V}}\right)$. This number has been used in studies by Armenio and Sarkar (2002) and Taylor et al. (2005) as an alternate stratification parameter.

$F r_{\mathrm{V}}^{2}=\frac{<w^{2}>}{N^{2} \cdot L_{\mathrm{E}}^{2}}=\left(\frac{L_{B}}{L_{E}}\right)^{2}=\frac{<w^{2}>}{\frac{g}{\rho_{0}} \cdot \rho_{r m s} \cdot L_{E}}=\frac{\mathrm{VKE}}{\mathrm{APE}}$

where $\mathrm{VKE}=\left\langle w^{2}>, L_{\mathrm{E}}=\rho_{\mathrm{rms}} \cdot\left|\left(\partial<\rho>(\partial z)^{-1}\right)^{-1}\right|\right.$ is the Ellison scale, $N^{2}=-g \cdot\left(\rho_{0}\right)^{-1} \cdot\left(\partial<\rho>(\partial z)^{-1}\right)$ is the Brunt-Väisälä frequency, and $L_{\mathrm{B}}=w_{\mathrm{rms}} N^{-1}$ is the buoyancy length scale. The root mean square values are denoted as $w_{\text {rms }}$ and $\rho_{\text {rms. }}$. The former length scale is an indicator of the work required to transport a fluid parcel a distance $L_{\mathrm{E}}$ from its equilibrium level, and the latter is a measure of the vertical displacement of a fluid parcel if its vertical kinetic energy were converted to potential energy. Hence, buoyancy becomes dynamically more important in the vertical mass transport compared to the work by shear-produced energetic turbulence, when $L_{\mathrm{B}}$ becomes less than $L_{\mathrm{E}}$. The square of the parameter $F r_{\mathrm{V}}$ can therefore be considered as a direct measure of the state of buoyancy on the turbulence structure itself.

The Ozmidov length scale has been employed in a number of studies on turbulence in stratified environments (Ozmidov 1965; Turner 1973; Ivey and Imberger 1991; Kay and Jay 2003). It is defined as $L_{\mathrm{O}}=\sqrt{ }\left(\varepsilon\left(N^{3}\right)^{-1}\right)$, with $\varepsilon$ the dissipation rate of turbulent kinetic energy. This length scale is derived from the energy density spectrum. It signifies the critical length scale, the smallest scale, at which buoyancy forces become important in the transfer of turbulent kinetic energy. A number of interpretations of this length scale exist. Monin and Obukhov (1953) and Ozmidov (1965) interpret this length scale as an indicator of the maximum length scale of isotropic turbulence in a stratified fluid. Above this length scale, the structure becomes anisotropic due to buoyancy forces. The horizontal motions are relatively unaffected by gravity, while the energy of vertical motions is both suppressed and redistributed to the horizontal motions.
Turner (1973) provided the following interpretation. It is the length scale at which the loss of energy due to buoyancy becomes comparable with that transferred or the length scale at which the buoyancy force is of the same order of magnitude as the inertial forces. According to Kay and Jay (2003), this length scale represents the vertical displacement of a fluid parcel if all of the turbulent kinetic energy would be converted to potential energy. Thus, the Ozmidov length scale is related to the turbulent kinetic energy balance rather than the buoyancy flux balance. Furthermore, due to buoyancy forces, the turbulent motions become more anisotropic. This means that the vertical turbulent kinetic energy is a better measure for the energy available by energetic turbulent structures to achieve vertical transport of fluid parcels than the total turbulent kinetic energy. Ivey and Imberger (1991) indicate that energetic turbulence cannot be maintained due to the combined effect of molecular viscosity $(\nu)$ and buoyancy forces when the turbulence intensity parameter $\varepsilon\left(\nu N^{2}\right)^{-1}<$ 15. Like the Ozmidov length scale, this parameter is related to the total turbulent kinetic energy balance rather than the vertical buoyancy flux balance. Consequently, the above considerations regarding the Ozmidov length scale apply for this parameter as well.

Our data show that when countergradient buoyancy fluxes occur, some turbulence kinetic energy is maintained. Hence, the effects of stratification on the vertical buoyancy flux balance are already appreciable before they affect the total kinetic energy balance. Therefore, here, we use turbulent quantities that determine the buoyancy flux balance (Eq. 3) directly to derive a discriminator parameter for the onset of vertical countergradient transport of an active scalar. Furthermore, we aggregate the effects associated with the redistribution by pressure correlations and dissipation in Eq. 3 into a relaxation term $\left(\varnothing_{\rho w}-\varepsilon_{\rho w}=\right.$ $\left.\left(\tau_{<\rho w>}\right)^{-1} \cdot<\rho w>\right)$

$$
\langle\rho w\rangle=\tau_{\langle\rho w\rangle}\left(-\left\langle w^{2}\right\rangle \frac{\partial\langle\rho\rangle}{\partial z}+\frac{A P E}{V K E}\left\langle w^{2}\right\rangle \frac{\partial\langle\rho\rangle}{\partial z}-\frac{\partial\langle\rho w\rangle}{\partial t}\right)
$$

in which $\tau_{<\rho w>}$ is a relaxation timescale. The first term on the right-hand side of Eq. 6 can be considered to represent ordinary Fickian diffusion (downgradient transfer), the second term can be considered a countergradient correction term which depends on the turbulent density variance or turbulent potential energy balance, and the third term a time derivative term which represents memory effects in the buoyancy flux balance. Note from Eq. 6 that the turbulent kinetic energy $\left(k=1 / 2\left(<u^{2}>+<v^{2}>+<w^{2}>\right)\right)$ does not need to be damped in order to produce a zero buoyancy flux, as would be required in the $k-\varepsilon$ type turbulent closure 
framework. Dimensional analysis of Eq. 6 with the assumptions of a quasi-steady state $\left(\left|\left(\tau_{\langle\rho w\rangle}\right)^{-1} \cdot\langle\rho w\rangle\right|>\mid \partial(\langle\rho w\rangle)\right.$. $\left.(\partial t)^{-1} \mid\right)$ and a relaxation timescale $\tau_{<\rho w>}$ equal to the absolute value of $S^{-1}=\left(\partial<U>\cdot(\partial z)^{-1}\right)^{-1}$ gives the following expression:

$\frac{\langle\rho w\rangle}{\frac{L_{B}}{L_{E}} \rho_{r m s} w_{r m s}}=\frac{\langle\rho w>}{-L_{B} \frac{\partial \rho}{\partial z} w_{r m s}} \equiv \sqrt{R_{i}}\left(1-\frac{1}{F_{\mathrm{RV}}^{2}}\right)$.

Note that the $R i$ number can be alternatively viewed as the four thirds power of the ratio of the Corrsin length scale (Smyth and Moun 2000) to Ozmidov length scale. The Corrsin length scale, defined as $L_{\mathrm{C}}=\sqrt{ }\left(\varepsilon\left(S^{3}\right)^{-1}\right.$, is considered to represent the smallest scale at which turbulent structures are deformed by the mean velocity shear. The mean velocity shear deforms turbulent structures, which causes the production of Reynolds stresses. These stresses, in combination with the mean shear, cause energy transfer from the main flow to the turbulent flow. Therefore, the kinetic energy and the range of scales of turbulent flow grow with increasing mean velocity shear. Equation 7 has been applied to the measurements obtained on April 14, 2005 and April 16, 2006. The results are shown in Fig. 5 in the following section and in Fig. 15 in Appendix 1.

\section{Results}

\subsection{General description of the turbulent flow characteristics}

The turbulence measurements with EMFs are presented in Fig. 3a. In Fig. 3b, mean flow data, collected with the upward- and downward-looking ADCPs, are presented. As a guide to the eye, salinity and SPM concentration measurements at $0.70 \mathrm{mab}$ have been included in Fig. $3 \mathrm{~b}$. It is observed that turbulence quantities (Fig. 3a), such as the turbulent kinetic energy (here $k=\left(<u^{2}>+<w^{2}>\right)$ ) and vertical turbulent transport of momentum, increase during the tidal filling phase (Fig. 3a, b, around 0550 hours) and during the passage of a SPM-laden salinity-induced density front (Fig. 3a, b, around 0800 hours; see also de Nijs et al. 2009). This indicates that turbulent mixing is active during both instances.

The peak in the SPM concentration measurements at 0800 hours is correlated with lower values of turbulent kinetic energy and vertical turbulent transport of momentum than at 0550 hours (Fig. 3a, c), even though the velocities are larger before than after 0800 hours. Although this indicates an increase of effective settling at 0800 hours, the observation of a three-layer system at station 6B (de Nijs et al. 2009) implies that this peak represents a combination of settling and advection of SPM.
The first panel of Fig. 3a shows a second distinct inflow event near the bed around 0800 hours. At the start of this event, even around the time when the current approximately reached its peak (before 0800 hours), the turbulence properties $k$ and $\langle u w\rangle$ are lower (0730-0800 hours) than at and after 0800 hours, when $k$ and $\langle u w\rangle$ show peaks. This corresponds with larger (overlying) SPM stratification near the bed before 0800 hours than at and after 0800 hours, which indicates damping of bed-generated turbulence SPMinduced buoyancy (around 0740 hours). Figure 4 (fourth panel at $0740-0800$ hours at $0.30 \mathrm{mab}$ ) shows that negative SPM fluxes occur at this time.

4.2 The observation of persistent countergradient transport fluxes

Figure 4 shows vertical turbulent transports of salinity (third panel 0700-0900 hours) and SPM with negative signs (fourth panel 0545, 0730-0800, and 0915 hours). These transports generally coincide with turbulent kinetic energy (first panel) and moment transfer (second panel). In the presence of stable ensemble mean stratification, negative signs depict turbulent mass transport against the concentration, i.e., the density gradient. It is noted that salinity and SPM stratification was always stable at 0.30 and $0.70 \mathrm{mab}$, but unstable salinity stratification (data not included here) events did occur at 3.20 mab (Fig. 4, around 0630 and 0730 hours). Stratification higher in the water column has been evaluated from measurements at 0.70 and $3.2 \mathrm{mab}$. The vertical turbulent transport of momentum is always with the gradient.

The majority of the negative transport flux events coincide with the passage of the density front (roughly between 0700 and 0930 hours). It should be noted that although tilt corrections altered the value of the mass fluxes, the corrections did not change the signs. Furthermore, negative salinity and SPM fluxes at a particular height do not always occur at the same time (Fig. 4, at $0.30 \mathrm{~m}$ above bed at 0545, 0730-0800, and 0945 hours). This indicates that the distribution of salinity and SPM fluctuations determines the signs. The turbulent statistics have been calculated with different ensemble periods as well. These showed consistent behavior when compared to each other and the persistent occurrence of countergradient buoyancy fluxes.

Countergradient SPM fluxes depict enhanced settling of SPM by fluid motions because an EMF measures velocity fluctuations which transport SPM. Velocity meters based on acoustic principles, however, do not measure the velocity of the fluid, but the velocity of the (individual) particles. Hence, their velocity fluctuation estimates contain some effects of the gravitational settling velocity of individual particles. Note that if turbulent fluctuations do not occur or 


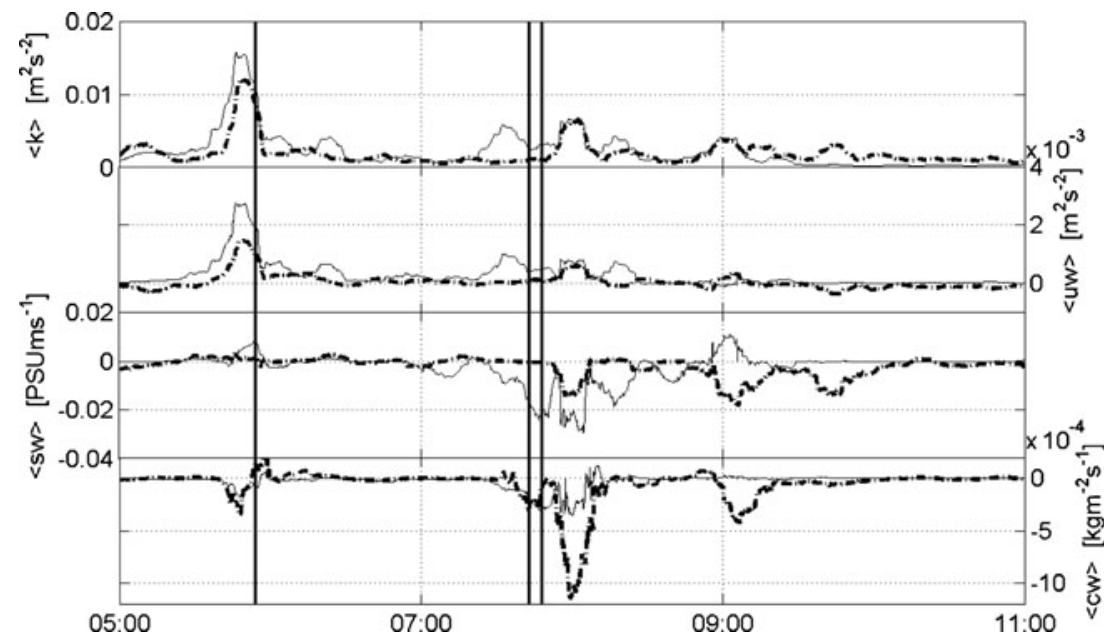

Fig. 4 Data recorded at the anchored station during the survey on April 14, 2005. From top to bottom: turbulent kinetic energy $(k=$ $\left.\left(<u^{2}>+<w^{2}>\right)\right)$, vertical turbulent transport of momentum $(<u w>)$, vertical turbulent transport of salinity $(\langle s w>)$, and vertical turbulent transport of SPM $(\langle c w\rangle)$, respectively. Time (hours) is shown on the $x$-axis. Data at $0.30 \mathrm{~m}$ (dashed thick line) and $3.20 \mathrm{~m}$ (thin line) above the bed are shown. The salinity stratification was unstable at $3.2 \mathrm{mab}$ around 0630 and 0730 hours. These events, associated with overturning motions, can explain the negative salinity induced buoyancy fluxes at that height are small, the EMF does not measure vertical SPM fluxes. However, SPM still settles due to the gravitational settling velocity of individual particles (Fig. 4, around 0730, 0845, and 0930 hours). Most of the SPM is located below the pycnocline near the bed (de Nijs et al. 2009) as a result of the effects of stratification on turbulence. This may explain the lack of correlation between negative SPM flux events at different levels.

\subsection{Application discriminator parameter}

In this section, the time development of $(R i)^{1 / 2} \cdot\left(F r_{\mathrm{V}}\right)^{-2}$ (Fig. 5b) is examined in relation to the sign of the buoyancy fluxes (Fig. 4, third and fourth panels) for the measurements collected on April 14, 2005. A similar analysis is done on the measurements collected on April 16, 2006 in Appendix 1 (Figs. 15, 16, and 17). The scaled buoyancy flux (Eq. 7 with $\rho=\rho_{0}+\gamma_{\mathrm{s}} \cdot S$ ) is shown in Figs. 5a and 15a in Appendix 1 as a scatter plot with $(R i)^{1 / 2} \cdot\left(F r_{\mathrm{V}}\right)^{-2}$ on the horizontal axis. The vertical lines in this figure denote the transition where buoyancy fluxes change sign. Figures $5 \mathrm{a}$ and 15a in Appendix 1 provide support for the importance of the relative balance $\mathrm{VKE}(\mathrm{APE})^{-1}$ on the behavior of the salinity-induced buoyancy flux. It can be seen from Fig. 5a that downgradient transports generally occur for $(R i)^{1 / 2}$. $\left(F r_{\mathrm{V}}\right)^{-2}<1$, while large countergradient transports mainly occur when $(R i)^{1 / 2} \cdot\left(F r_{\mathrm{V}}\right)^{-2}>2$. This threshold value is about 2 for $\left((R i)^{1 / 2} \cdot\left(F r_{\mathrm{V}}\right)^{-2}\right)_{C}$ (not shown) based on SPM-induced buoyancy (the subscript $C$ denotes $\rho=\rho_{0}+\gamma_{\mathrm{c}} \cdot C$ ). The threshold values 1 and 2 for salinity-induced buoyancy fluxes are plotted as horizontal lines in Fig. 5b. From this figure, it is inferred that generally, salinity-induced buoyancy creates an imbalance between turbulent potential and vertical kinetic energy (APE $>$ VKE, $\left.\left((R i)^{1 / 2} \cdot\left(F r_{\mathrm{V}}\right)^{-2}\right)>1-2\right)$, while SPM-induced buoyancy is in approximate balance $\left(\mathrm{APE}<\mathrm{VKE},\left((R i)^{1 / 2} \cdot\left(F r_{\mathrm{V}}\right)^{-2}\right)_{C}<2\right)$. This result may not be surprising since the supply or availability of SPM is limited and settling of SPM acts to reduce $\mathrm{APE}_{\mathrm{C}}$.

From a comparison of Figs. 4 (third panel) and 5b, the statistically significant values of $(R i)^{1 / 2} \cdot\left(F r_{\mathrm{V}}\right)^{-2}$ above $1-2$ around 0515, 0700, 0800, 0900, and 0940 hours (Fig. 5b) can be seen to correlate with the observations of countergradient salinity fluxes (Fig. 4, third panel). Around 0550, 0620, 0640, and 0740 hours, values of $(R i)^{1 / 2} \cdot\left(F r_{\mathrm{V}}\right)^{-2}$ are below 1 (Fig. 5b). These values coincide with the observations of downgradient salinity fluxes at these times (Fig. 4, third panel). The statistically significant values of $\left((R i)^{1 / 2} \cdot\left(F r_{\mathrm{V}}\right)^{-2}\right)_{C}$ above 2 around 0605, 0740, 0800, and 0915 hours (Fig. 5b) coincide with the observations of countergradient SPM fluxes (Fig. 4, fourth panel). Around 0555, 0620, 0855, and 0940 hours, values of $\left((R i)^{1 / 2}\right.$. $\left.\left(F r_{\mathrm{V}}\right)^{-2}\right)_{C}$ are below 1 (Fig. 5b). These values are consistent with the observation of downgradient SPM fluxes (Fig. 4, fourth panel). The values of $\left((R i)^{1 / 2} \cdot\left(F r_{\mathrm{V}}\right)^{-2}\right)_{C}$ remain close to the threshold between 0550 and 0600 hours because $R i$ appears to be overestimated when compared to $R i_{\mathrm{f}}$ (see de Nijs and Pietrzak, in prep). Hence, the measurements collected on April 14, 2005 and April 16, 2006 show a similar consistent behavior regarding the relation between statistical significant values of $(R i)^{1 / 2} \cdot\left(F r_{\mathrm{V}}\right)^{-2}$ (Figs. $5 \mathrm{~b}$ and $15 \mathrm{~b}$ in Appendix 1) and change of sign of the buoyancy fluxes (Figs. 4 and 16 in Appendix 1, third and fourth 


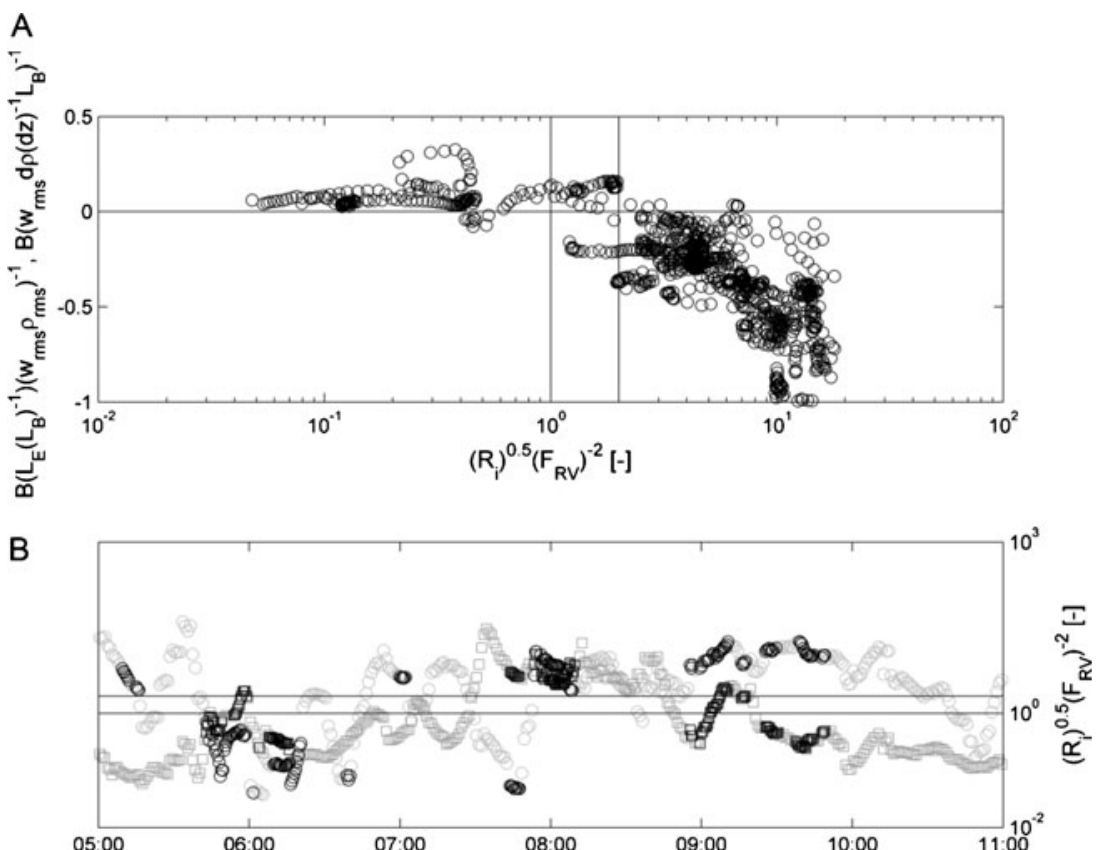

Fig. 5 Turbulence measurements recorded at the anchored station obtained at April 14, 2005 (about $22 \mathrm{~h}$ of turbulence measurements). a Graph showing the dimensionless salinity-induced buoyancy flux vs. $\left((R i)^{1 / 2} \cdot\left(F r_{\mathrm{V}}\right)^{-2}\right)$. The vertical lines denote, from left to right, threshold values of 1 and 2. b Graph showing time series of $\left((R i)^{1 / 2} \cdot\left(F r_{\mathrm{V}}\right)^{-2}\right)$ indicated by the open circles and $\left((R i) \cdot\left(F r_{\mathrm{V}}\right)^{-2}\right)_{C}$ indicated by the open

panels), i.e., parameter values above the threshold correlate with countergradient buoyancy flux events and parameter values below it with downgradient fluxes.

In a number of cases, countergradient salinity- and SPM-induced buoyancy flux events are correlated (Fig. 4, third and fourth panels near the bed around 0800, 0910, and 0945 hours). This suggests that the salinity-induced buoyancy determines the countergradient transport of SPM. However, the measurements acquired on April 14, 2005 show that at 0550 and 0740 hours, values of $\left((R i)^{1 / 2} \cdot\left(F r_{\mathrm{V}}\right)^{-2}\right)_{C}$ and $\left((R i)^{1 / 2} \cdot\left(F r_{\mathrm{V}}\right)^{-2}\right)$ are above and below their thresholds, respectively (Fig. 5b). Furthermore, around these time instances, salinity buoyancy fluxes are positive and small (Fig. 4, third panel). Therefore, SPM-induced buoyancy appears to determine the countergradient SPM transports. However, between 0540 and 0550 hours, values of $\left((R i)^{1 / 2}\right.$. $\left.\left(F r_{\mathrm{V}}\right)^{-2}\right)_{C}$ are below the threshold of 2. This may indicate the importance of $\langle c s\rangle$ in the balance equation of the SPM buoyancy flux, which would result in higher values of $\left((R i)^{1 / 2} \cdot\left(F r_{\mathrm{V}}\right)^{-2}\right)_{C}$.

The relative importance of salinity- and SPM-induced buoyancy is further elucidated using the transport equation of the vertical turbulent SPM flux and SPM concentration variance, and the assumption that salinity and SPM are active and passive scalar quantities, respectively. We substitute $\rho=\rho_{0}+\gamma_{\mathbf{c}} \cdot C+\gamma_{\mathbf{s}} \cdot S$ in Eqs. 3 and 4 and neglect squares. The horizontal lines denote threshold values of 1 and 2. It is noted that the calculated $(R i)^{1 / 2} \cdot\left(F r_{\mathrm{V}}\right)^{-2}$, which had disputable statistical significance, were left out of the analysis at first. This resulted in much loss of data. For the purpose of analysis, these data have been included in the graph indicated by the gray color, while statistically significant data are depicted by the black color

time and spatial derivatives of the salinity and SPM crosscorrelation terms

$$
\begin{aligned}
\frac{\partial\left\langle\gamma_{c} c w\right\rangle}{\partial t}-\frac{\partial w_{s}\left\langle\gamma_{c} c w\right\rangle}{\partial z}= & -\left\langle w^{2}\right\rangle \frac{\partial\left\langle\rho_{0}+\gamma_{c} c\right\rangle}{\partial z}-\frac{g}{\rho_{0}} \\
& \times\left(\left\langle\left(\gamma_{c} c\right)^{2}\right\rangle+\left\langle\gamma_{\mathrm{cs}} c s\right\rangle\right) \\
& +\phi_{\langle\gamma c w\rangle}-\varepsilon_{\langle\gamma c w\rangle}
\end{aligned}
$$

$$
\begin{aligned}
\frac{\partial\left\langle\left(\gamma_{\mathrm{c}} c\right)^{2}\right\rangle}{\partial t}-\frac{\partial w_{s}\left\langle\left(\gamma_{c} c\right)^{2}\right\rangle}{\partial z} & = \\
& -2\left\langle\gamma_{\mathrm{c}} c w\right\rangle \frac{\partial\left\langle\rho_{0}+\gamma_{\mathrm{c}} C\right\rangle}{\partial z}-\varepsilon_{\left\langle\left(\gamma_{c} c\right)^{2}\right\rangle}
\end{aligned}
$$

in which $\gamma_{\mathrm{cs}}=\gamma_{\mathrm{c}} \cdot \gamma_{\mathrm{s}} ; w_{\mathrm{s}}$ is the settling velocity of SPM; $\phi_{<\gamma_{c} \mathrm{cw}}>$ depicts the redistribution of $\left\langle\gamma_{\mathrm{c}} \mathrm{c} w\right\rangle$ by pressure fluctuations; and $\varepsilon_{<\gamma_{c} \mathrm{cw}>}$ and $\varepsilon_{<\left(\gamma_{c} c\right)^{2}>}$ are the dissipation rates of $\left\langle\gamma_{c} c w\right\rangle$ and $\left\langle\left(\gamma_{c} c\right)^{2}\right\rangle$, respectively.

The second and third terms on the right-hand side of Eq. 8 are the buoyancy terms. The behavior of these terms will be discussed for the 2005 measurements at 0.30 mab. It can be seen from Fig. 6a that values for $\left\langle\left(\gamma_{s} s\right)^{2}>\right.$ are generally larger than $\left\langle\gamma_{\mathrm{cs}} c s>\right.$ and from Fig. $6 \mathrm{~b}$ that values for $\left\langle\gamma_{\mathrm{cs}} c s\right\rangle$ are generally larger than $\left\langle\left(\gamma_{\mathrm{c}} c\right)^{2}\right\rangle$. However, from about 0540 to 0600 hours, $<\left(\gamma_{\mathrm{c}} c\right)^{2}>\approx<\gamma_{\mathrm{cs}} c s>$, while 
Fig. 6 Turbulence measurements collected on April 14, 2005 recorded at the anchored station. a Graph showing < $\left(\gamma_{\mathrm{s}} s\right)^{2}>($ black $)$ and $<\gamma_{\mathrm{cs}} c s>$ (gray). b Graph showing < $\left(\gamma_{\mathrm{c}} c\right)^{2}>($ black $)$ and $<\gamma_{\mathrm{cs}} c s>$ (gray)
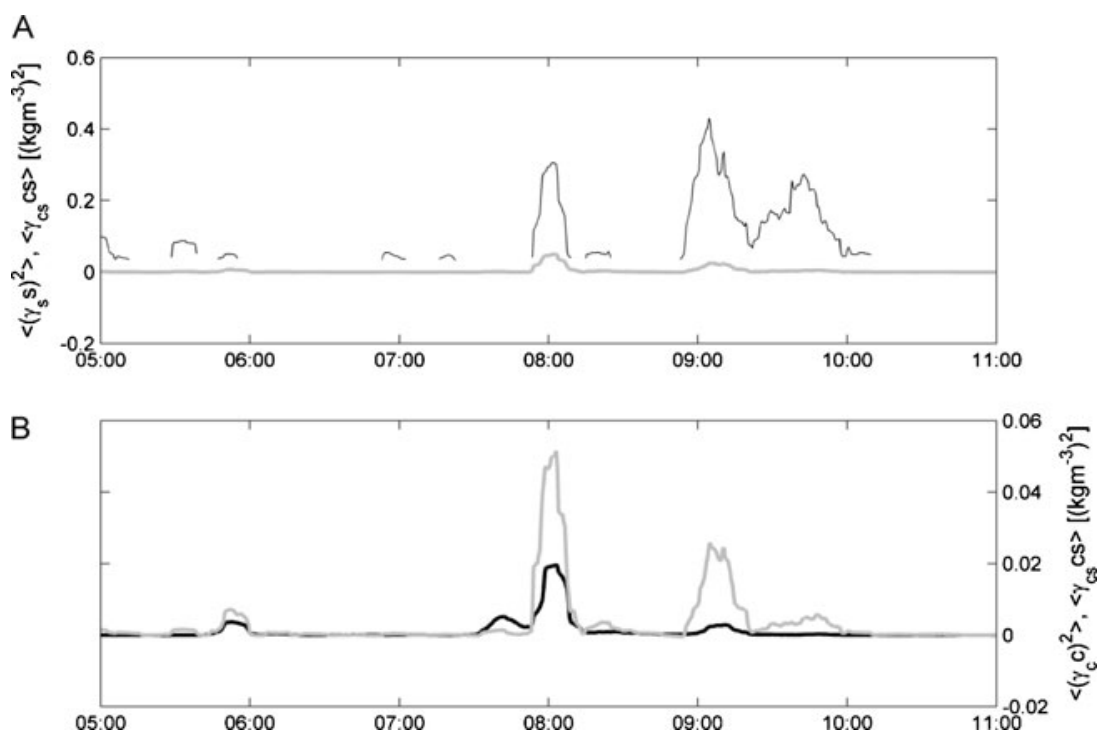

around 0740 hours, values for $\left\langle\left(\gamma_{\mathrm{c}} c\right)^{2}>\right.$ are larger than $<\gamma_{\text {cs }} c s>$ (Fig. 6b). Therefore, this supports the view that SPM descends to the bed mainly by salinity-induced buoyancy-driven motions, while around 0740 hours, SPM-induced buoyancy is the dominant driving force. The buoyancy terms $\left\langle\left(\gamma_{\mathrm{c}} c\right)^{2}>\right.$ and $\left\langle\gamma_{\mathrm{cs}} c s>\right.$ in Eq. 8 cause differences in turbulence diffusivities and turbulent Prandtl-Schmidt numbers between salinity and SPM because the turbulent energetic mixing term is proportional to the ensemble mean density gradient. The term $\left\langle\gamma_{\mathrm{cs}} c s\right\rangle$ represents the interaction between the distribution of salinity (active scalar) and SPM (passive scalar). SPM is less affected by salinity-induced buoyancy when SPM and salinity are less correlated or uncorrelated. Hence, then SPM becomes the active scalar. The majority of the

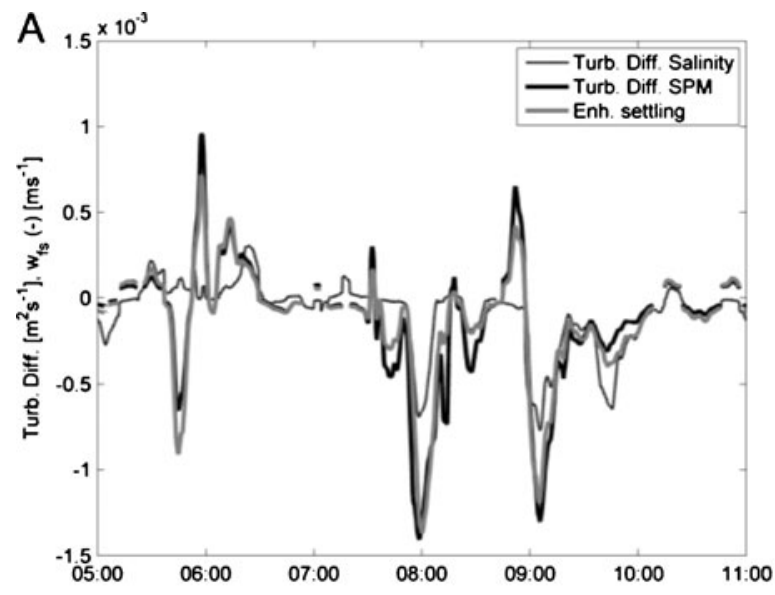

Fig. 7 Turbulence measurements collected on April 14, 2005 recorded at the anchored station. a Graph showing time series of estimates of turbulence diffusivities for salinity and SPM and estimates of fluid countergradient buoyancy fluxes are induced by salinityinduced density variance. This can be explained by an additional dissipation of SPM-induced buoyancy flux and density variance as a result of settling (see the second term on the left-hand side in Eqs. 8 and 9).

In order to establish the significance of the countergradient buoyancy fluxes, estimates for the turbulence diffusivities of salinity and SPM are determined. In order to do this, the gradient-type transport assumption is applied, even though this assumption is violated when countergradient fluxes occur. These estimates are presented in Fig. 7a, b. The absolute values of the diffusivities for downgradient buoyancy fluxes (Fig. 7a, around 0550 hours) and for countergradient buoyancy fluxes (Fig. 7a, around 0740 and 0800 hours) are of the same order of

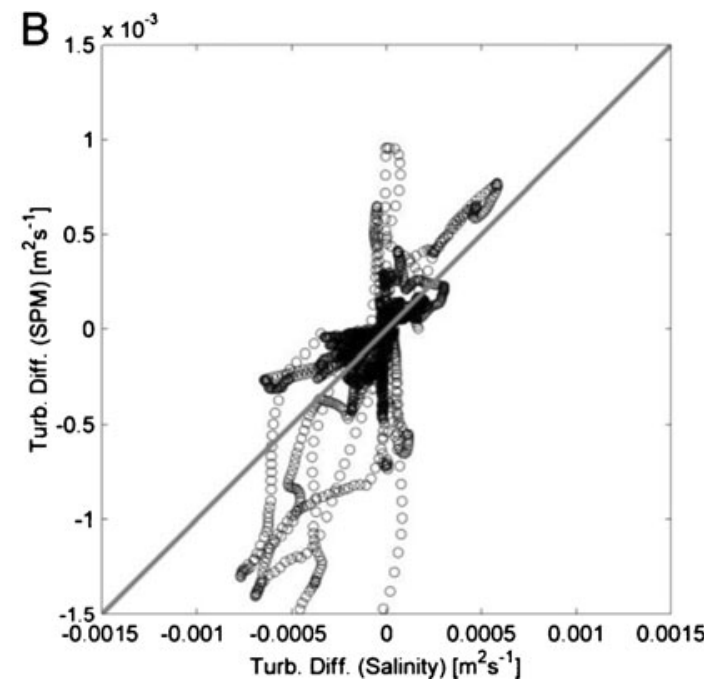

motion-induced settling velocities. b Graph showing a scatter plot of the turbulence diffusivity for salinity vs. the turbulence diffusivity for SPM. The solid line indicates perfect correlation 
magnitude, and they are orders of magnitude larger than the molecular diffusivity.

In order to investigate the significance of the SPM-induced countergradient buoyancy fluxes, we then compare the fluid motion-induced settling velocity (referred to as enhanced settling in Fig. 7a) with the gravitational settling velocity. Figure 7 shows the enhanced settling which has been calculated by dividing the buoyancy flux with the local ensemble mean SPM concentration. This enhanced settling is of the same order as settling velocities measured in this part of the estuary (MKO 1983) and reported by other studies (van Leussen 1994). Similar conclusions can be drawn from Fig. 18 in Appendix 2. Figure 7b shows that the diffusivities for salinity and SPM do not perfectly correlate, which is expected based on the effect of the second term on the right hand-side in Eq. 8 (see discussion above).

\subsection{Intermittent motions in the turbulent flow}

In this section, the nature of the turbulence- and buoyancyinduced motions is discussed in more detail. Gerz and Schumann (1996) presented conceptual models (Fig. 8a, b) of the contribution of different scales to countergradient transport of heat and momentum in homogeneous stratified shear flows based on LES data and measurements. In the following, an interpretation is given on how to apply their conceptual model to inhomogeneous turbulent shear flow conditions.
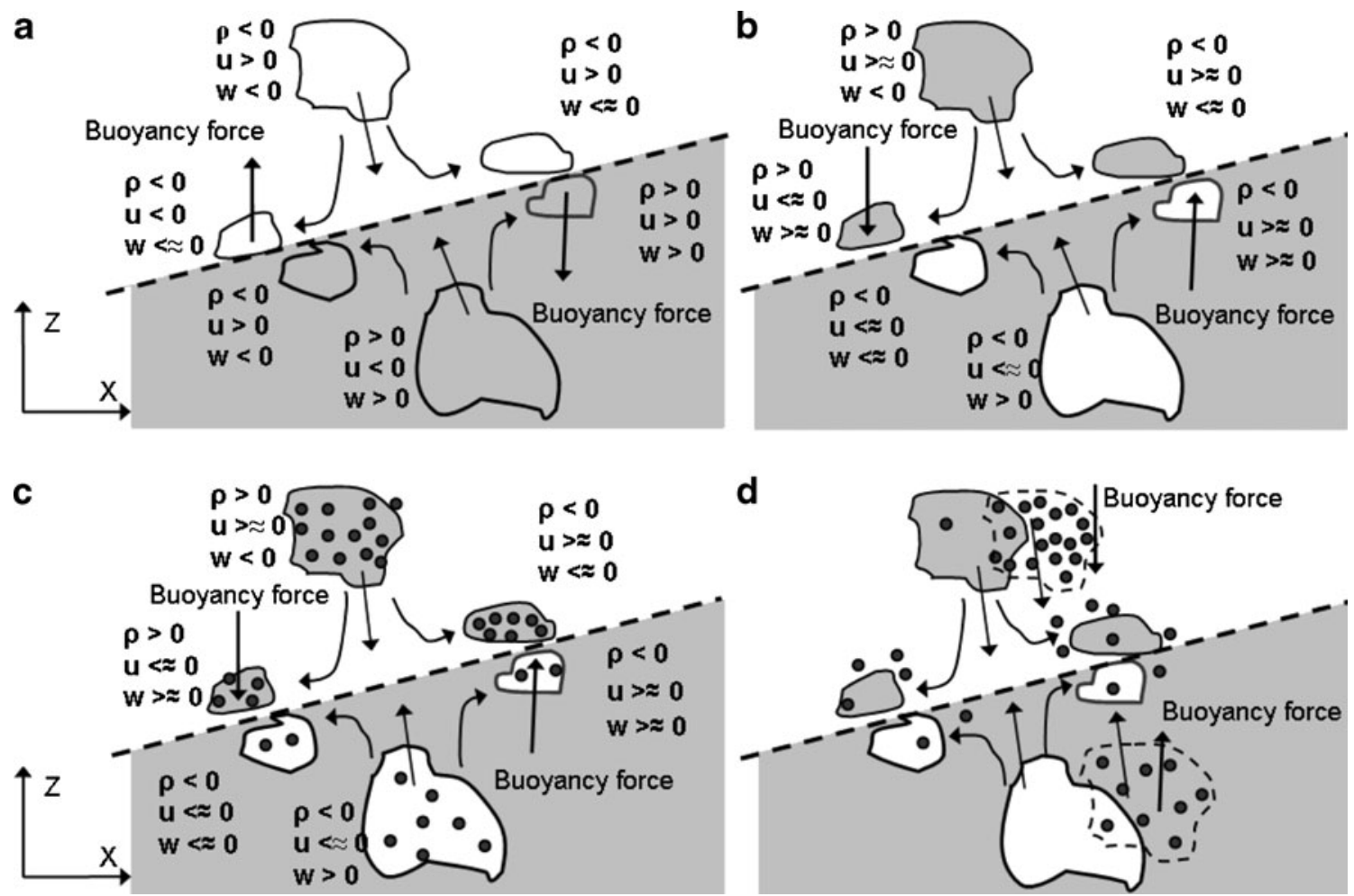

Fig. 8 a, b Conceptual sketches redrawn from Gerz and Schumann (1996) of the intermittent motions which govern the turbulent structure, momentum, and mass transfer for moderately and highly stratified turbulent conditions, i.e., conditions comprising stably stratified flows controlled by shear or affected by buoyancy (a) and strongly stratified flows with weak shear $(R i \approx 0.5-1)$ or controlled by buoyancy (b). SPM has been included in $\mathbf{c}$ and $\mathbf{d}$ (indicated by the dots). $\mathbf{c}$ Graph showing transports and collisions of parcels of fluid driven by salinity-induced buoyancy that contain relatively low SPM concentration levels. d Graph showing transport and collisions of fluid parcels by salinity- and SPM-induced buoyancy. Panel a shows the occurrence of countergradient transport at small scales, while $\mathbf{b}-\mathbf{d}$ also depict countergradient transport at large scales. Panel $\mathbf{c}$ shows that the salinity-induced buoyancy determines the transport of SPM (passive scalar), while d also depicts the contribution of SPM-induced buoyancy (active scalar). a Parcels of fluid advected through the legs of hairpin vortices (not drawn) colliding in the frontal or convergence zone set up by pairs of overlaying head-up and head-down hairpin vortices. The frontal zone is depicted by the tilted straight line. Momentum is exchanged through pressure and mass through mixing when fluid parcels collide. Parcels of higher density will sink and resist rising induced by energetic turbulent structures, while lighter fluid parcels will rise and resist forced sinking by energetic structures. Due to asymmetry in transport of the smaller fluid parcels along the collision or convergence, front countergradient buoyancy fluxes develop at the small scales. b Turbulent motions in the regime $R i \approx$ $0.5-1$ (hence $R i>R i_{\mathrm{cr}}$ ). These turbulent motions are determined by incompletely mixed fluid parcels which move by their own buoyancy. These fluid parcels are remnants of past energetic transport events which move back to their equilibrium levels in terms of density. They rise or descend and therefore may occasionally collide with each other. Collisions cause them to break up into smaller fluid parcels; such events can be considered to represent contributions to mass and momentum transfer at smaller scales 


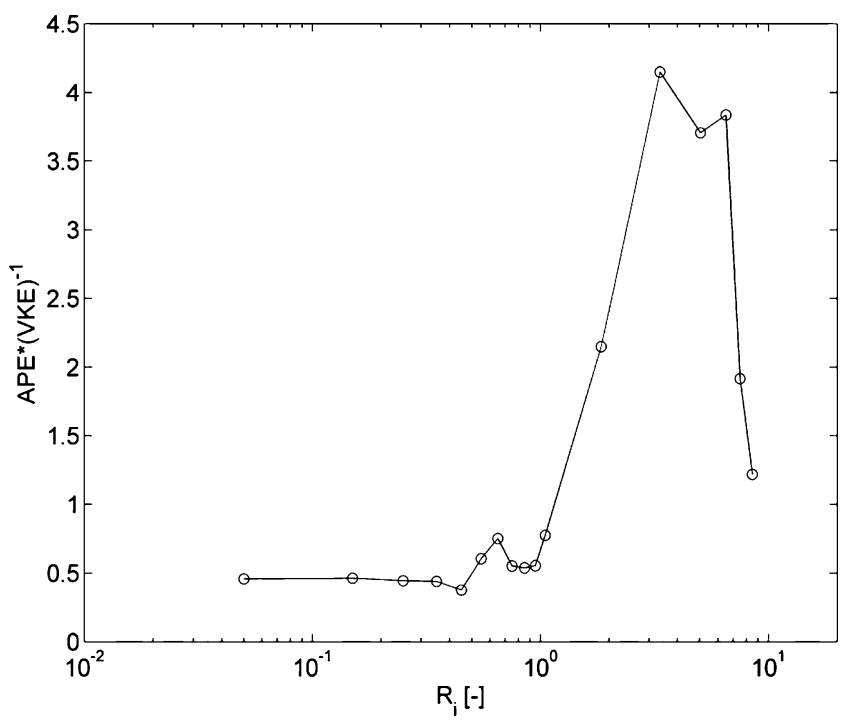

Fig. 9 Turbulence measurements collected on April 14, 2005 recorded at the anchored station. The graph shows binned values for $\mathrm{APE}(\mathrm{VKE})^{-1}$ vs. Ri. Note, however, that this ratio is not uniquely described by $R i$

From a phenomenological viewpoint, countergradient flux can be explained by incompletely mixed or dissipated parcels of rising water of lower density and descending water of higher density, which have retained a certain memory of their mixing history or turbulent properties. This memory lasts longer for salinity-induced density fluctuations than for velocity fluctuations because momentum can be transferred more quickly with the surrounding fluid through pressure fluctuation forces than mass which requires mixing. It is anticipated that the memory effects induced by the density fluctuations increase with $R i$ due to damping of mixing processes. This can be investigated by plotting the ratio of APE to VKE vs. Ri (Figs. 9 and 19 in Appendix 3). Figure 7 shows that this ratio increases with $R i$ until it reaches a maximum. This trend can be ascribed to differences in decay rates between APE and VKE, i.e., VKE decays faster than APE because positive buoyancy fluxes convert VKE to APE. However, at large $R i$, the ratio of APE to $\mathrm{VKE}$ decreases. This tendency can be explained by an increase of the decay rate of APE and a decrease of the decay rate of VKE due to the conversion of APE by countergradient buoyancy fluxes to $\mathrm{VKE}$ in order to restore some kind of equilibrium between APE and VKE. This would explain why the turbulent Prandtl-Schmidt numbers can become larger than 1 and increase with $R i$ (de Nijs and Pietrzak, in prep).

The application of the principle elements that form the conceptual model by Gerz and Schumann (1996) is presented here: (1) an asymmetry in the transport of fluid parcels at the small scales determined by the effects of buoyancy on transport by energetic turbulent structures and (2) buoyantly moving fluid parcels representing incompletely mixed remnants of past energetic transport events, as an explanation of countergradient transport along the convergence or frontal zone, do not necessarily need to be limited to homogeneous turbulent conditions. The energy of the large-scale countergradient motions at large $R i$ is supplied by conversion of potential to kinetic energy at the large or countergradient scale, while the energy supplied to the countergradient motions at the small scales stems from energy from the production at large scales. Therefore, this principle also holds for non-homogeneous turbulent conditions. However, it must be considered whether the collision principles apply. Furthermore, the integrated effect of transport of fluid parcels by turbulent energetic structures needs to be taken into account rather than the contribution by (individual) pairs of hairpin vortex packets. It must be considered that fluid advected upward between the legs of a head-up hairpin vortex does not necessarily collide with fluid advected downward by headdown vortices. Therefore, collisions can occur with noncoherently moving fluid masses. In the case of strong stratification, the explanation for countergradient transfer does not alter for inhomogeneous conditions since remnants of past energetic mixing events are driven by buoyancy. Therefore, their dynamics do not directly rely on the energetic conditions and motions.

Recent studies in inhomogeneous sheared flows show evidence of a coherent ordering of hairpin vortices inclined to the wall into groups termed packets (Fig. 10a). The resulting flow pattern induced by the arrangement of hairpin vortex packets is shown in Fig. 10b. A convergence zone can be identified as for homogeneous conditions (Fig. 8), but here it is set up by Q2 and Q4 events driven by an arrangement of head-up hairpin vortices, contrary to the convergence zone set up for homogeneous conditions by pairs of overlying head-up and head-down vortices.

The sketch in Fig. 11 shows how buoyancy effects would favor the importance of quadrants outward and inward interactions with increasing $R i$ as found in the measurements. Furthermore, near the bed head-up, vortex structures occur more often, hence the up-going motions induced by these structures. Therefore, in terms of past mixing events, it can explain why down-going motions which contain more salinity and SPM determine the countergradient flux at 0.30 mab (see next section). Their signature in the measurement records is further investigated with quadrant and spectral analyses.

4.5 Investigation of the intermittent motions with quadrant decomposition and spectral analysis

Next, records are examined using quadrant analysis of $u$, $w$ and $c, w$ (Table 1 and Fig. 12). The effects of buoyancy on transport by energetic structures and the presence of buoyancy-driven motions would increase the importance of outward and inward interaction quadrants compared to the 
a

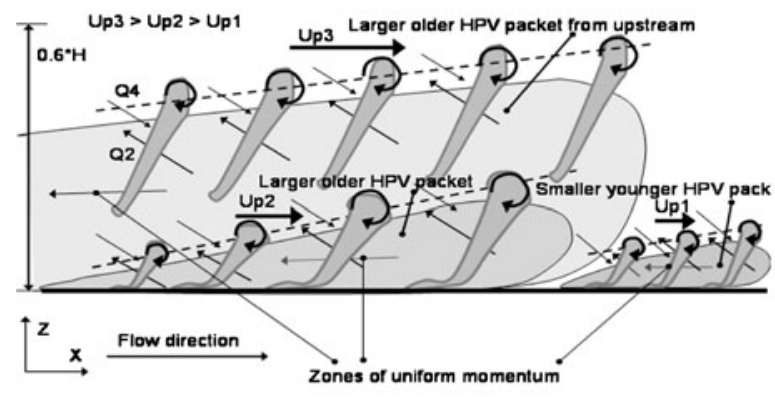

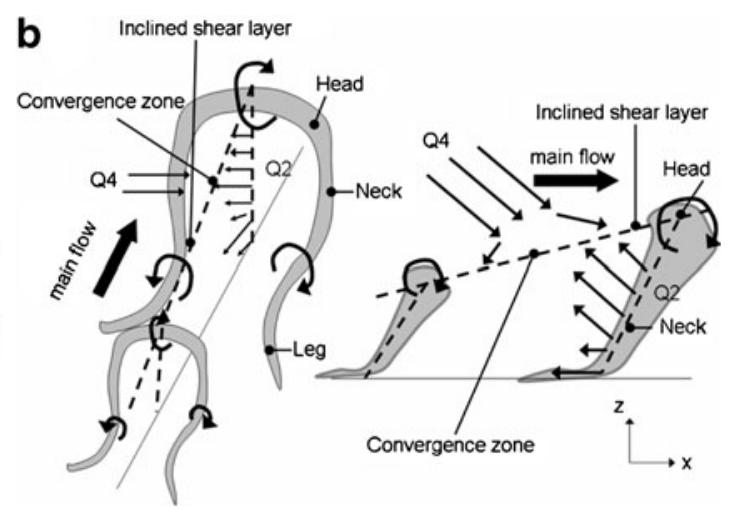

multiple uniform momentum zones and lower velocity toward the wall. The vertical extent of these structures, or packets, is about $0.6 \mathrm{H}$, with $H$ the water depth, and the streamwise length is about $1.5 H$ (Natrajan and Christensen 2006). The size of the individual hairpin vortices varies. The hairpin packet model proposed by Adrian et al. (2000) indicates that hairpin packets form a dynamical arrangement of similar structures whose dimensions increase with distance from the wall. This model provides a possible basis for numerous turbulent structural features such as low-speed streaks, uniform momentum zones, and the presence of large-scale structures throughout the boundary layer. b Conceptual sketch illustrating the transfer of mass and momentum by organized hairpin vortices (packets) in a ramp-like structure within the log layer of wall turbulence. Most of the transfer occurs in the region of opposing Q2 and Q4 events (Natrajan and Christensen 2006)

signs. It can be seen from Table 1 (columns 6 and 7) that at 0550 hours, the upward motions (0.67) determine the fractional distribution to $\langle c w\rangle$ while downward motions determine the fractional distribution to $\langle u w\rangle$. At 0740 hours, upward motions determine $\langle u w\rangle$ and downward motions $\langle c w\rangle$, while at 0748 hours, downward motions determine both $\langle u w\rangle$ and $\langle c w\rangle$.

The aforementioned findings differ from results obtained by Nezu and Nakagawa (1993), Kawanisi and Yokosi (1993), and Nikora and Goring (2002) for equilibrium-neutral conditions. Their results show that ejection and sweep events (here second and fourth quadrants) dominate both the fractional time $(60 \%)$ and contribution to $\langle u w\rangle$ and $\langle c w\rangle$. A similar distribution is only observed at 0550 hours (Table 1 , second row). This observation indicates that the turbulent structure is (slightly) affected by buoyancy effects at this time. Quadrant analysis of field measurements by West and Oduyemi (1989) showed that for neutral conditions, sweep and ejection events make up about $59 \%$ of the fractional time of $\langle u w\rangle$, but for more stratified conditions, this value decreased to $52 \%$, implying a more even distribution of the time fraction over the quadrants. West and Shiono (1988) and Dyer et al. (2004) deduced similar tendencies from quadrant analysis of velocity and salinity, and velocity and SPM data, respectively. Numerical results (3D LES) by Taylor et al. (2005) also support the previously described trends in $\langle u w\rangle$. the record at 0550 hours, $\langle c w\rangle$ estimates have negative 


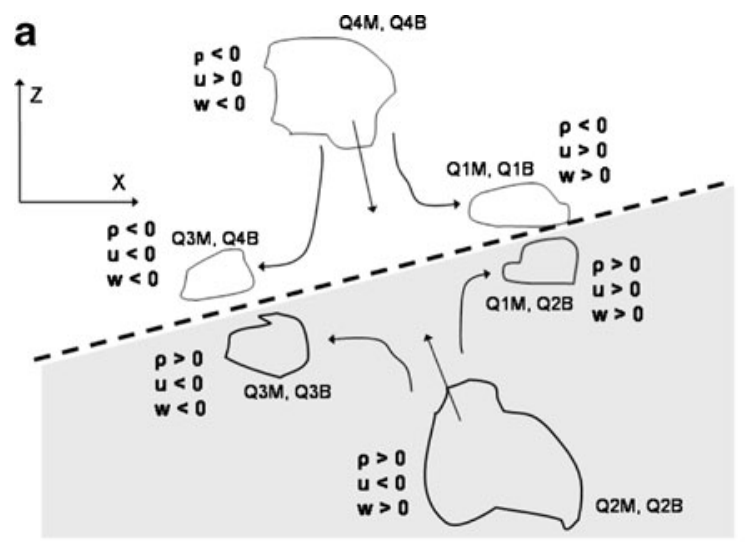

Fig. 11 a, b Convergence front at a inclined shear layer depicted by the dashed line where flows convergence and hence parcels of fluid collide due to advection by opposing Q2 and Q4 events at the large scales. These events are induced by energetic turbulent structures or packets of hairpin vortices. At the convergence front, Q1 and Q4 events form at the small scales, which contributes to the momentum flux (Q1M, Q4M), and associated Q1, Q2, Q3, and Q4 events develop at the small scales, which contributes to the buoyancy flux (Q1B, Q2B, Q3B, and Q4B). Here, we use the quadrant decomposition by $\mathrm{Lu}$ and Willmarth (1973) for positive main flow $(<U>>0)$ as Q1 $(u>0$, $c<0, w>0$; outward interaction), Q2 $(u<0, c>0, w>0$; ejection), Q3 ( $u$ $<0, \mathrm{c}>0, w<0$; inward interaction), and Q4 ( $u>0, c<0, w<0$; sweep). In both graphs, countergradient contributions at the smaller scales can be discerned, even though the momentum and buoyancy flux averaged over all quadrants depict downgradient transport. The graphs illustrate conditions where buoyancy forces play minor and major roles on the parcels of fluid transported by energetic turbulent structures, respectively. For conditions resembling strong stratification (b), the Q4 and Q2 events at the large scales are suppressed compared to those portrayed for weak stratification (a). Furthermore, the individual Q1, Q2, Q3, and Q4 events at the small scales in b may travel and transport $(u, c)$ further vertically before being remixed with their surroundings than in a. Hence, with increasing stratification contribu-

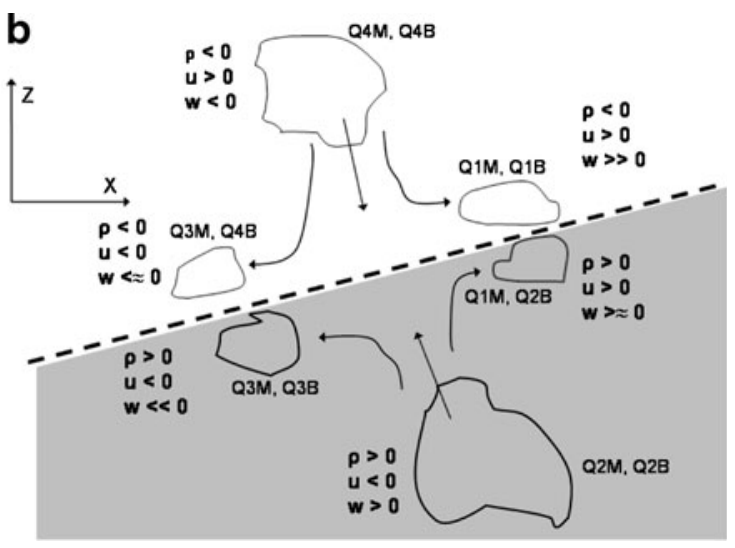

tions by Q1, Q2, Q3, and Q4, events at the smaller scales may become relatively more important in the exchange of momentum and mass at the expense of Q2 and Q4 events at the large scales. With increasing stratification, an asymmetry in transport develops at the smaller scales, which reduces the mean downgradient transport over all quadrants, i. e., the average of the quadrants at the smaller scales in a results in $\langle u w\rangle=0,\langle c w\rangle=0$ (a) and in $\langle u w\rangle>0,\langle c w\rangle\langle 0$ (b). This indicates that the distribution of the fractional time and contribution over the quadrants of $u w, s w$, and $c w$ becomes more even from weak to strong stratification. Note that the downgradient buoyancy flux is more susceptible to changes in stratification which induce damping of the mean downgradient transports and countergradient transports than the downgradient momentum flux. This is because the Q2 and Q4 events which produce positive buoyancy fluxes at the smaller scales (a) become less relevant with increasing stratification at the expense of Q1 and Q3 events (b) which produce negative buoyancy fluxes. This also explains turbulent Prandtl-Schmidt numbers larger than 1 and their increase with $R i$ (de Nijs and Pietrzak, in prep). Note that the sketches $(\mathbf{a}, \mathbf{b})$ represent the integrated effect of advection induced by turbulent structures or packets, i.e., collisions of fluid parcels do not necessarily take place as a result of overlying turbulent structures but can collide with incoherently moving fluid. The convergence zone is therefore not necessarily a fixed and well-defined feature
Fig. 12 Quadrant analyses applied to 10-min records of velocity fluctuations $(u, w)$ and SPM concentration fluctuations (c) around 0740 hours at $0.30 \mathrm{~m}$ above bed. a Graph showing $u$ vs. $w$. b Graph showing $c$ vs. $w$. The labels in the quadrants depict fractional time and contribution, respectively (see Eq. 1). Note that around 0740 hours, the vertical turbulent transport of salinity (Fig. 4) does not show any substantial variation and that its values remain close to zero
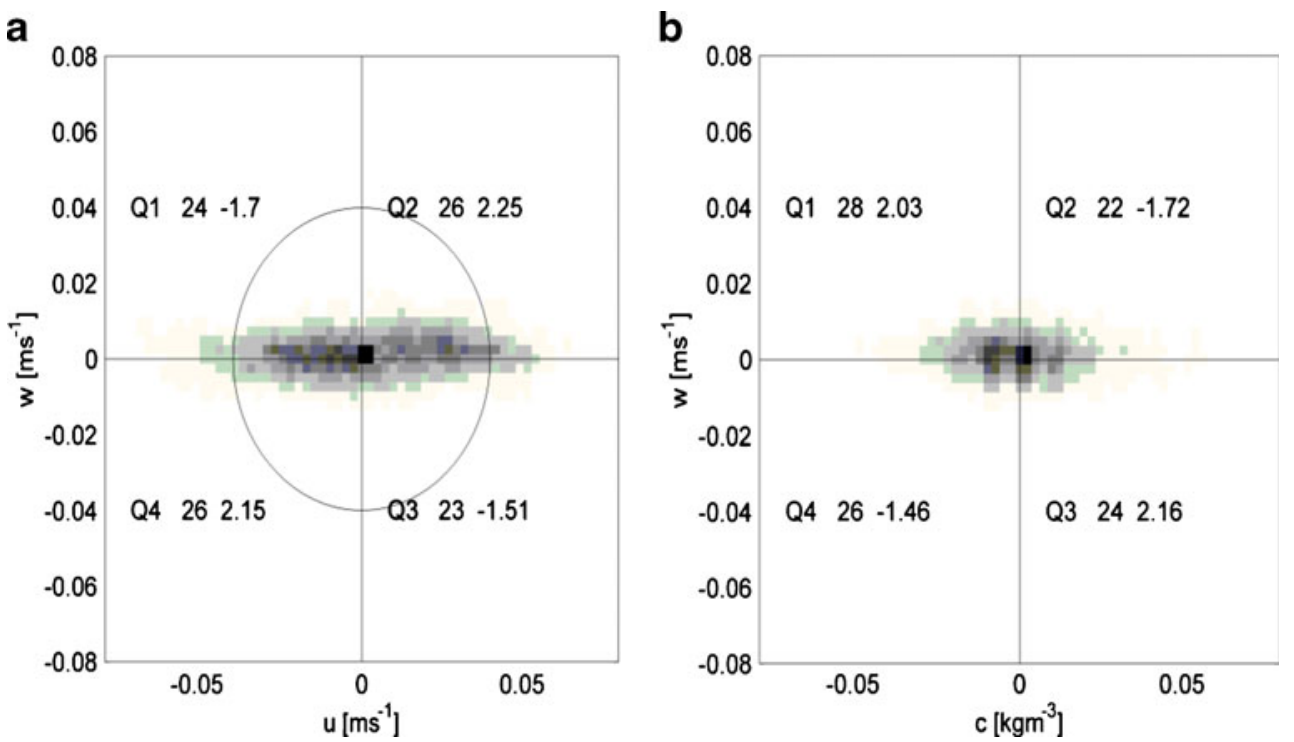
Table 1 shows that around 0550 hours, a distinct ejection and sweep structure can be identified representative for (slightly) buoyancy-affected and neutral conditions where upward motions determine $\langle c w\rangle$ (Figs. 8a and 11). At other times, down-going motions determine $\langle c w\rangle$ and fractional contributions are about evenly distributed over the quadrants, indicative of conditions when the turbulent structure is dynamically controlled by buoyancy forces (Figs. 8a, b and 11). When the turbulence structure is dominated by buoyancy forces (at 0740 hours), the magnitudes of the fractional contributions in all quadrants are greater than for buoyancy-affected turbulence (around 0550 hours). This distribution has also been observed by West and Oduyemi (1989) and West and Shiono (1988). Thus, the quadrant analysis of $\langle u w\rangle$ indicates that the record at 0740 hours at 0.30 mab exhibits a distinctly different structure and intensity of the turbulent motions than for buoyancyaffected conditions (at 0550 hours). The quadrant classification applies to downgradient $\langle c w\rangle$, when it is determined by energetic turbulent motions. Quadrant analysis indicates that down-going motions determine countergradient $\langle c w\rangle$, which corresponds with the conceptual sketches presented in Figs. 8 and 11. Note that when tidal mixing was active (around 0550 hours), up-going motions (ejections) determined $\langle c w\rangle$. These motions can serve as an explanation in terms of past mixing events for the down-going motions that determine $\langle c w\rangle$ at 0740 hours. Furthermore, the motions which determine $\langle u w\rangle$ and $\langle c w\rangle$ are different. Hence, it indicates that knowledge of the type of motions which determine $\langle u w\rangle$ is not a replacement for that of $<c w>$.

Studies by West and Shiono (1988) and West and Oduyemi (1989) have attributed low values of $\langle s w\rangle$ and $\langle c w\rangle$ to internal wave events. Dyer et al. (2004) and Taylor et al. (2005) associate their occurrence with countergradient $\langle c w\rangle$ and $\langle\rho w\rangle$ values. Spectral analysis (of $c, w$ and $s, w$ ) has therefore been applied to the whole record (Fig. 13) and to 10 -min records at $0.30 \mathrm{~m}$ above bed at 0550 and 0740 hours (Fig. 14). The measurements collected on April 16, 2006 are examined in Appendix 4 (Figs. 20 and 21). The spectral analysis indicates that most of the covariance is contained in the low-frequency motions, but shows the occurrence of countergradient transport at small scales as well. However, distinct phase differences $(|\Delta \varphi|)$ near $1 / 2 \pi$ indicative of internal wavelike behavior could not be distinguished. In fact, a wide range of phase relationships exists, indicative of various types of motions. Figure 13 shows $C_{\mathrm{osw}},|\Delta \varphi|_{\mathrm{sw}}$ and $C_{\mathrm{ocw}}, \mid$ $\left.\Delta \varphi\right|_{\mathrm{cw}}$ as a function of time and frequency. It can be seen from the upper graphs that most of the covariance $(\langle s w\rangle$, $\langle c w\rangle)$ is contained at the low frequencies $\left(<0.1 \mathrm{~s}^{-1}\right)$. The lower graphs show that at these frequencies $\left(<0.1 \mathrm{~s}^{-1}\right)$, just before 0550 hours, $|\Delta \varphi|$ is between 0 and $1 / 2 \pi$ (white and gray colors), which indicates mixing of salinity and SPM by energetic structures. After 0740 hours, $|\Delta \varphi|$ is between $1 / 2 \pi$ and $\pi$ (gray and black colors) in the range of negative buoyancy fluxes, which depicts countergradient transport.

Figure $14 \mathrm{a}, \mathrm{b}$ shows the co-spectra of $u$ and $w, s$ and $w$, and $c$ and $w$ at 0.30 mab at 0500 hours $(R i<0.25)$ and 0740 hours $(R i>>0.25)$. Positive and negative values in the cospectra and phase angles between $0 \leq|\Delta \varphi|<1 / 2 \pi$ and $1 / 2 \pi$ $<|\Delta \varphi| \leq \pi$ depict downgradient and countergradient fluxes, respectively. It can be seen from Fig. 14a that when stratification is weak, $R i<0.5$ values of the co-spectra are generally positive and phases between $0 \leq|\Delta \varphi|<1 / 2 \pi$, particularly at the large scales of motion where most of the energy is contained. The aforementioned values are indicative of active mixing by turbulence (Fig. 14a, third to sixth panels). However, at smaller scales, some negative values of small magnitude in the co-spectra and phases between $1 / 2 \pi<|\Delta \varphi| \leq \pi$ indicative of countergradient fluxes are observed.

From Fig. 14b (at 0740 hours), it can be seen that when stratification is strong, $R i>>0.25$ values of the co-spectra and phases are also negative and between $1 / 2 \pi<|\Delta \varphi| \leq \pi$ at the large-scale energetic motions. Hence, these values indicate that buoyancy becomes dynamically active in the vertical turbulent transport. Therefore, it depicts conversion of potential energy into turbulent kinetic energy by buoyancy induced motions (Fig. 14b, third to sixth panels). At both time instances (Fig. 14a, b, around 0550 and 0740 hours), phase angles near $|\Delta \varphi| \approx 1 / 2 \pi$ associated with internal waves occur. However, they mostly coincide with small magnitude values in the co-spectra. Hence, internal waves if present hardly contribute to the turbulent fluxes.

\section{Discussion}

Measurements obtained from two separate deployments of an anchored station, in a low energetic stratified environment, show persistent countergradient vertical turbulent salinity and SPM transports. These countergradient fluxes took place during periods of stable ensemble mean salinity and SPM stratification. The parameter $(R i)^{1 / 2} \cdot\left(F r_{\mathrm{V}}\right)^{-2}$, which incorporates the relative balance between available potential energy and vertical turbulent kinetic energy, can predict their occurrence. This balance represents the ratio of the potential strength of buoyancy-driven motions to the counteracting work by the (large-scale) energy-containing turbulent motions. Buoyancy forces generally resist work done by energetic turbulent structures. Therefore, this alternate discriminator parameter indicates the onset of convective motions, that 
Fig. 13 Co-spectral density (first panel of $\mathbf{a}, \mathbf{b}$ ) and absolute phase angles (second panel of $\mathbf{a}, \mathbf{b}$ ) of salinity (a) and SPM (b) fluctuations with vertical velocity fluctuations as a function of time (hours) and frequency calculated from data at $0.30 \mathrm{mab}$ collected at the anchored station on April 11, 2005. The black lines denote time instances ( 0550 hours and $R i<0.5,0740$ hours and $R i \approx 10$ ) where co-spectral density and absolute phase angles of $\langle u w\rangle$, $\langle s w\rangle$, and $\langle c w\rangle$ have been plotted as line graphs (Fig. 14)
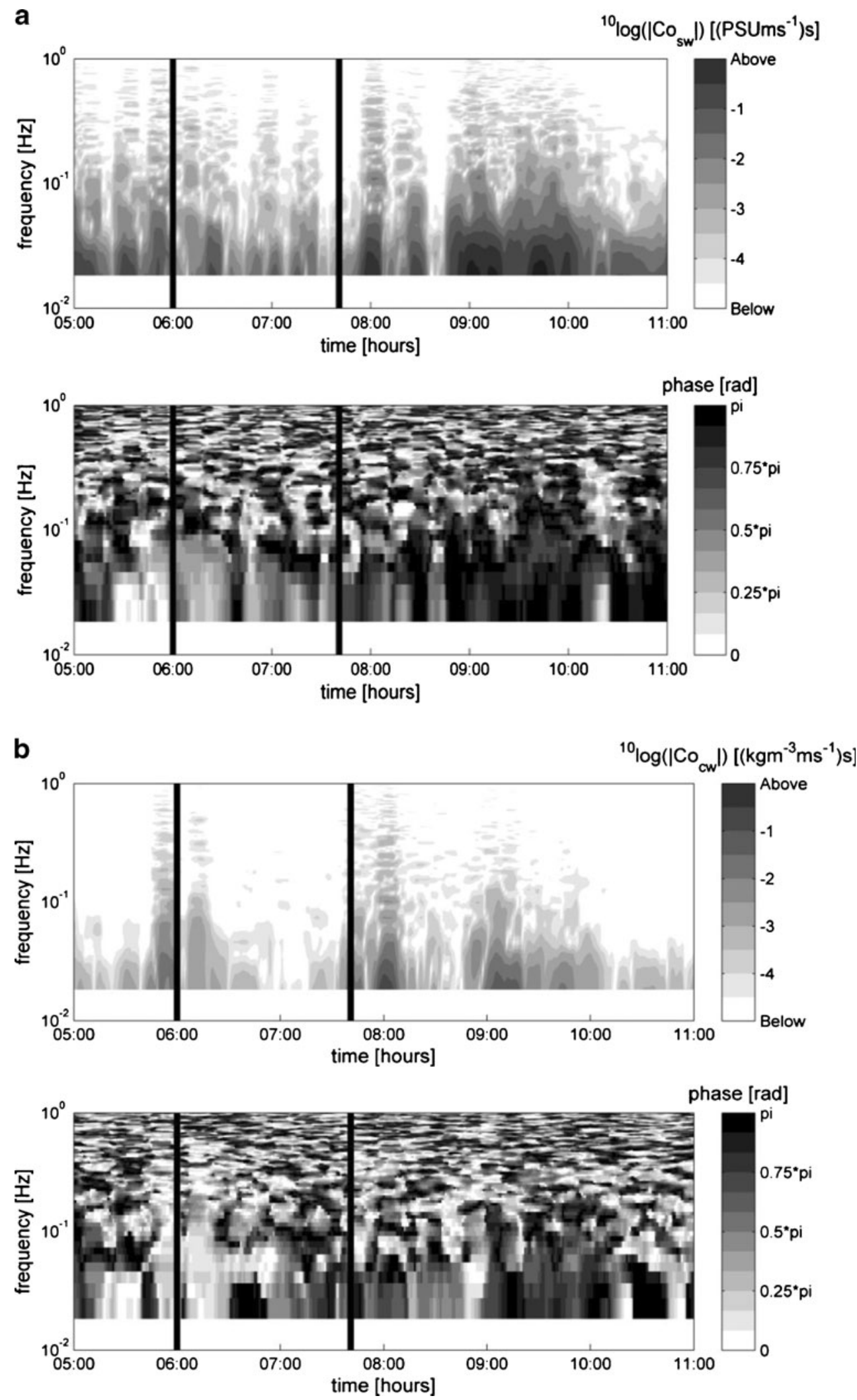
Fig. 14 a, b From top to bottom: normalized co-spectral density and absolute phase angles of $u$ and $w, s$ and $w$, and $c$ and $w$ as a function of $k(=$ $\left.\left(<U>\cdot f^{-1}\right)^{-1}\right)$ calculated from measurements collected on April 14,2005 at $0.30 \mathrm{mab}$ at 0550 hours (a) $(R i<0.5)$ and at 0740 hours (b) $(R i>>0.5)$. Note that around 0740 hours, the vertical turbulent transport of salinity does not show any substantial variation and that its values remain close to zero (Fig. 4, third panel) a

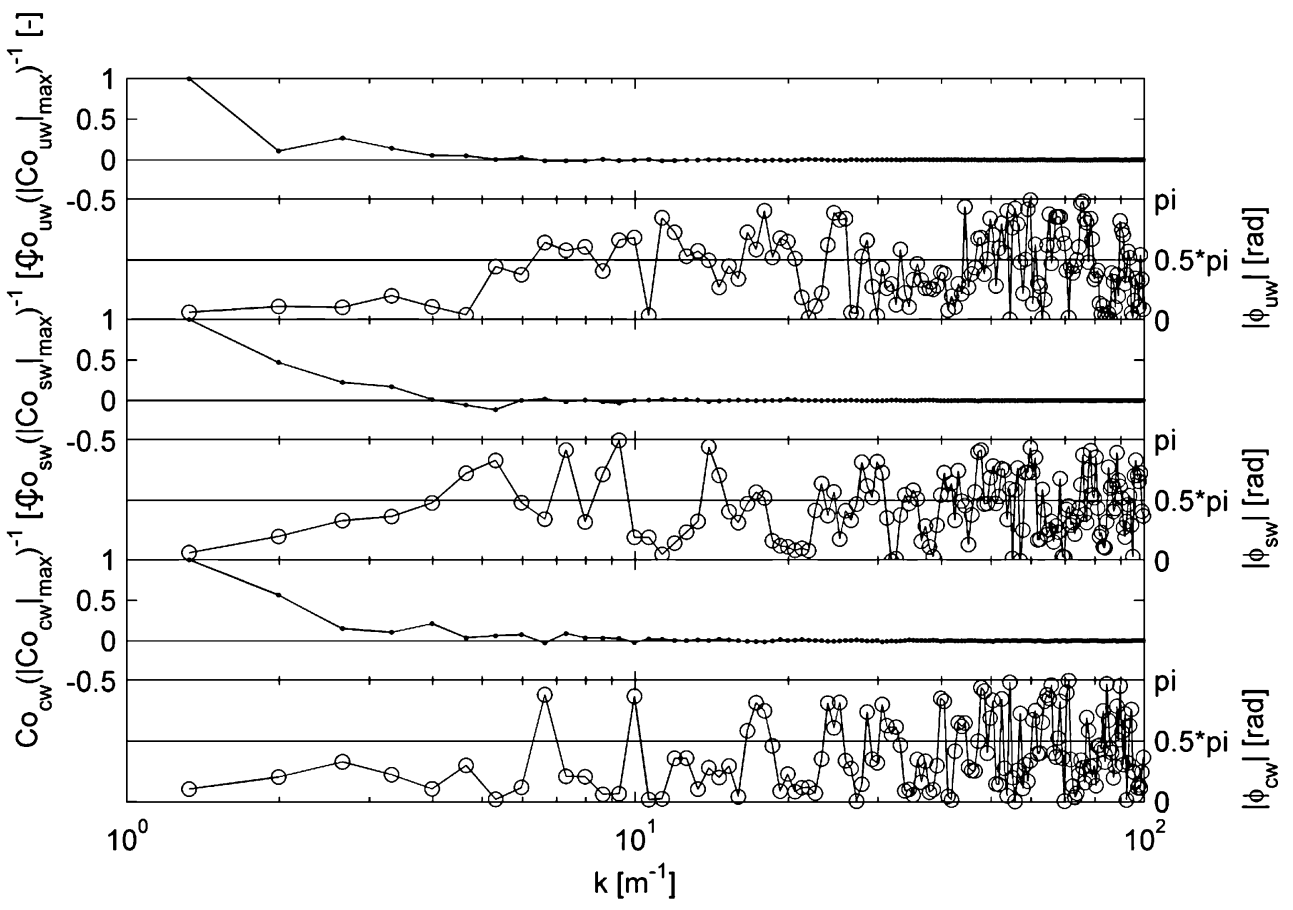

b

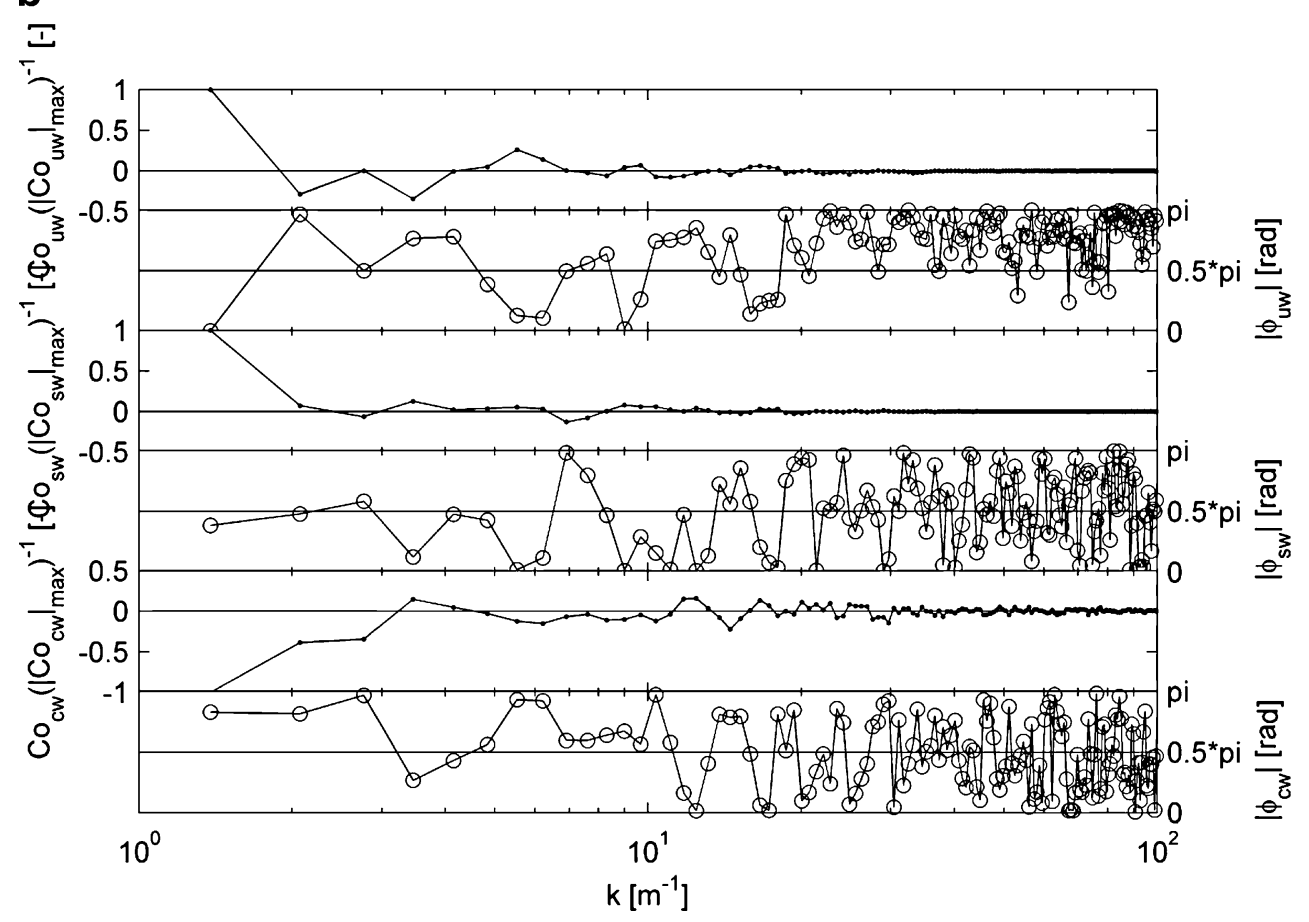

is, when buoyancy actively determines the vertical transport of mass. Mathematically, the countergradient buoyancy fluxes represent a conversion of APE, in the form of density variance, into VKE.

The spectral analysis shows the expansion of countergradient fluxes at moderate to small wavenumbers with increasing $R i$. The quadrant analysis shows a more even distribution of the fractional time and contribution over the quadrants with increasing $R i$. These observations are in agreement with the conceptual model by Gerz and Schumann (1996) of the effect of buoyancy forces on the transport induced by energetic structures and transport by convective motions in homogeneous flows. At small and intermediate scales, for low to moderate stratification, they 
represent asymmetry in the transport of fluid parcels by energetic turbulent structures after their breakup during collisions in convergence zones (Figs. 6a and 9). At the large scales and at large $R i$, they represent parcels of heavier and lighter fluid than their surroundings; they are remnants of transport by energetic structures in the past (Fig. 6b-d). The dominant flow structures are buoyantly moving fluid parcels which return to their equilibrium position in terms of density. This memory effect is likely to be ascribed to fluid parcels that are not completely remixed or broken down. Therefore, countergradient transport can be considered to result from motions which are associated with reversible contributions of transport by energetic turbulent structures (in the past). Hence, these motions depict a conversion of APE into VKE.

The countergradient buoyancy fluxes are associated with processes of restratification (sharpening of gradients) and enhanced settling of SPM. In the majority of the countergradient flux cases, $\left\langle\gamma_{\mathrm{cs}} c s>>><\left(\gamma_{\mathrm{c}} c\right)^{2}\right\rangle$ and $\left\langle\left(\gamma_{\mathrm{s}} s\right)^{2}\right\rangle>><$ $\left(\gamma_{c} c\right)^{2}>$. Therefore, SPM predominantly descends in salinity-induced buoyancy-driven motions toward the bed. However, at 0740 hours, $<\left(\gamma_{\mathrm{c}} c\right)^{2}>>><\gamma_{\mathrm{cs}} c s>$ and $\left\langle\left(\gamma_{\mathrm{c}} c\right)^{2}>\right.$ $>><\left(\gamma_{s} s\right)^{2}>$, which indicates that SPM-induced buoyancy motions also play a role. This is supported by the observation that negative SPM and salinity flux events do not always correlate.

Consequently, we have identified new mechanisms which contribute to the SPM trapping efficiency of harbor basins at the salt-freshwater interface. However, it is anticipated that this SPM trapping mechanism can also be applied to more general stratified bodies of water, such as lakes, estuaries, and shelf seas. For instance, the effects of buoyancy forces on turbulence are found to maintain an ETM at the head of the salt wedge in the Rotterdam Waterway (de Nijs et al. 2010b). Large stratification and decoupling effects associated with turbulence damping have been observed at the pycnocline (de Nijs et al. 2010a, b). Hence, the mechanisms that determine countergradient buoyancy fluxes can contribute to the salt balance and trapping of SPM at the fresh-saltwater interface and ETM region in estuaries as well.

The increase of the importance of the quadrants outward and inward interactions, relative to the quadrants ejections and sweeps, has been attributed by other investigators to internal waves (e.g., West and Oduyemi 1989; West and Shiono 1988) or their effects on the turbulence structure (Dyer et al. 2004). The reduction of the momentum, salinity, and SPM transport correlation coefficients with increasing $R i$, has been attributed to (short period) internal wavelike phenomena. The internal waves cause $u, s$, or $c$ to be approximately $1 / 2 \pi$ out of phase $(|\Delta \varphi|)$ with $w$ (West and Shiono 1988; West and Oduyemi 1989; Taylor et al. 2005) and $u$ to be $\pi$ out of phase $(|\Delta \varphi|)$ with $s$ and $c$ (West and Shiono 1988; West and Oduyemi 1989). 3D LES results by Taylor et al. (2005) showed the occurrence of countergradient buoyancy fluxes in the pycnocline region of an open-channel flow. They also associated these fluxes with internal waves. Dyer et al. (2004) measured countergradient $\langle c w\rangle$ in and above a lutocline layer, which they ascribed to enhanced settling of SPM due to the production of inactive turbulence and its interaction with the concentration profile. They attributed this turbulence structure to the effects of internal waves on the turbulent bursting structure.

However, some studies indicate that buoyancy forces induce a different dynamic response and geometrical structure on turbulence. These forces tend to make the turbulence structures more anisotropic and quasi-2D (e.g., Monin and Obukhov 1953; Ozmidov 1965; Riley and LeLong 2000; Armenio and Sarkar 2002; Taylor et al. 2005; Davidson 2004). This may be substantiated further by results of Venayagamoorthy and Stretch (2006). They investigated mixing and dispersion in decaying stratified turbulence. Their results indicate that under strong stratification, particle movements are restricted to the horizontal plane, while for low $R i$, particles move three-dimensionally. Furthermore, time histories of vertical velocity and density fluctuations of particles indicated that the density and vertical velocity fluctuations become about $1 / 2 \pi$ degrees out of phase for strongly stratified conditions. Therefore, they concluded the emergence of internal wave behavior.

The spectral analysis presented here shows a wide range of phase relationships. This is indicative of the coexistence of a wide range of types of turbulent and convective motions. Buoyancy may force a more quasi-2D nonisotropic motion upon the structure of turbulent motions themselves. The development toward a more quasi-2D turbulent structure may appear in single-point measurements as absolute phase differences between the vertical velocity and SPM fluctuations between $1 / 2 \pi$ and $\pi$, but with phase differences $(|\Delta \varphi|)$ closer to $1 / 2 \pi$. Therefore, transport associated with these turbulent structures may have an internal wavelike signature in single-point records of turbulence measurements, but is not necessarily the result of internal waves. Instead, Fig. 8 indicates that the turbulent structure is governed by different motions. This suggests that explanations for countergradient buoyancy fluxes or a decrease in correlation coefficients of vertical turbulent transports of mass with stratification in terms of internal waves (pumping, decay) or the effects of internal waves on the bursting structure are not required.

An explanation for the phase relationship behavior for $\rho$ and $w$, consistent with our analysis, is a shift in the relative importance between energetic turbulent structures and buoyancy-driven motions in the transport of mass. Therefore, different regimes can be distinguished based upon 
their relative contribution to vertical turbulent mass transport. When vertical shear dominates and the flow is mildly affected by buoyancy forces, phase differences between $\rho$ and $w$ at the large scales are expected to have values between $0 \leq|\Delta \varphi| \leq 1 / 2 \pi$. This depicts the dominance of energetic turbulent structures in the downgradient transport of mass. However, buoyancy forces suppress vertical motions by converting kinetic energy into potential energy. This may inhibit the lifting and stretching of coherent vortices in coherent structures or packets and subsequently reduce the effectiveness of the exchange of mass by ejection and sweep events. Phase differences around $1 / 2 \pi$ would depict considerable resistance by buoyancy forces on the transport by energetic structures and, hence, a significant reduction of the downgradient mass transport. At the small scales, countergradient buoyancy fluxes develop (Figs. 8 and 11) associated with phase differences between $1 / 2 \pi \leq|\Delta \varphi| \leq \pi$. When the flow becomes controlled by buoyancy forces, buoyancydriven motions become the dominant structures at the expense of turbulent energetic structures (Figs. 8 and 11). Obviously, these intermittent motions interact. However, buoyancy becomes dynamically more significant in the transfer of mass than the effects of shear and energetic turbulent structures, resulting in phase differences at the large scales between $1 / 2 \pi \leq|\Delta \varphi| \leq \pi$, which depict countergradient transport. The energy transfers for the different regimes are discussed in an accompanying paper (de Nijs and Pietrzak, in prep).

In most 3D numerical models, diffusivities for SPM and salinity are taken equal. However, it can be deduced from the balance equation of the vertical turbulent transport of a passive scalar (e.g., Eq. 8) that eddy diffusivities for passive (SPM) and active scalars (salinity) not only depend on their mean gradients but also inherently differ due to the work by the buoyancy correlation terms (Fig. 6). The measurements confirm this. For the dynamical significance of SPM-induced buoyancy in the vertical transport of mass, the intermittent distributions of salinity and SPM are of importance (Fig. 8c, d). Here, it can determine whether SPM predominantly acts as a passive or active scalar.

The measurements indicate that indirect methods to estimate buoyancy fluxes are not generally applicable in stratified environments. For instance, some studies employ the Osborn-Cox method to estimate buoyancy fluxes from microstructure profiles. This method assumes a balance between the buoyancy flux and diffusive dissipation of temperature or density variance only (Eq. 9). Furthermore, nowadays, it has become common practice to estimate Reynolds shear stresses from ADCP measurements in the field using the variance method. However, the majority of the ADCP configu- rations only allow for Reynolds shear stress estimates. This study indicates the importance of the combined knowledge of $\operatorname{VKE}\left(\left\langle w^{2}>\right)\right.$ and the interaction with the buoyancy field indicated by APE $\left(g<\rho^{2}>\left(\rho_{0} \partial \rho(\partial z)^{-1}\right)^{-1}\right)$. Hence, the measurements show that ADCP Reynolds stress measurements in combination with turbulent Prandtl-Schmidt number relations alone cannot serve as substitute estimates for measurements of vertical turbulent buoyancy fluxes.

Acknowledgments This research is supported by the Dutch Technology Foundation STW, Applied Science Division of NWO, and the Technology Program of the Ministry of Economic Affairs. The authors would like to thank Han Winterwerp for his support. The authors thank the Port of Rotterdam, in particular Herman Meijer, for giving support to this research. The authors also thank RWS Directorate Zuid-Holland, in particular Ad Schipperen and Arie Barendrecht, for providing vessels to deploy the rig. We would also like to thank the EU 6th Framework Programme project ECOOP, "European Coastal Sea Operational Observing and Forecasting System" for providing support for the preparation of this paper.

Open Access This article is distributed under the terms of the Creative Commons Attribution Noncommercial License which permits any noncommercial use, distribution, and reproduction in any medium, provided the original author(s) and source are credited.

\section{Appendix 1}

Application discriminator parameter

In this appendix, the time development of $(R i)^{1 / 2} \cdot\left(F r_{\mathrm{V}}\right)^{-2}$ for the measurements collected on April 16, 2006 are discussed. It is convenient to note that the current near the bed is determined by tidal filling between 1615 and 1730 hours and by the passage of a density current between 1700 and 2100 hours. The parameter $\left((R i)^{1 / 2} \cdot\left(F r_{\mathrm{V}}\right)^{-2}\right)$ is plotted in Fig. 15a, b in Appendix 1. The scatter plot of scaled buoyancy flux (Fig. 15a in Appendix 1) indicates similar threshold values to Fig. 5a, i.e., downgradient and countergradient transports occur when $\left((R i)^{1 / 2} \cdot\left(F r_{\mathrm{V}}\right)^{-2}\right)<1$ and $\left((R i)^{1 / 2} \cdot\left(F r_{\mathrm{V}}\right)^{-2}\right)>2$, respectively. Figure $15 \mathrm{~b}$ in Appendix 1 shows statistical significant values of $\left((R i)^{1 / 2} \cdot\left(F r_{\mathrm{V}}\right)^{-2}\right)$ above the thresholds 1-2 between 1600-1610, 1700-1715, 1815-1830, and 1930-2000 hours (Fig. 15b in Appendix 1), which is consistent with observations of countergradient salinity fluxes at these times (Fig. 16, third panel, in Appendix 1). Values of $\left((R i)^{1 / 2} \cdot\left(F r_{\mathrm{V}}\right)^{-2}\right)$ below the thresholds 1-2 occur around 1610 hours and between 1645 and 1700 hours. These values are in agreement with the observation of downgradient salinity fluxes (Fig. 16, third panel, in Appendix 1, around 1610 hours and between 1645 and 1700 hours).

Downgradient SPM buoyancy fluxes (Fig. 16, fourth panel, in Appendix 1) are observed between 1800 and 1815 hours, which is consistent with statistically signifi- 


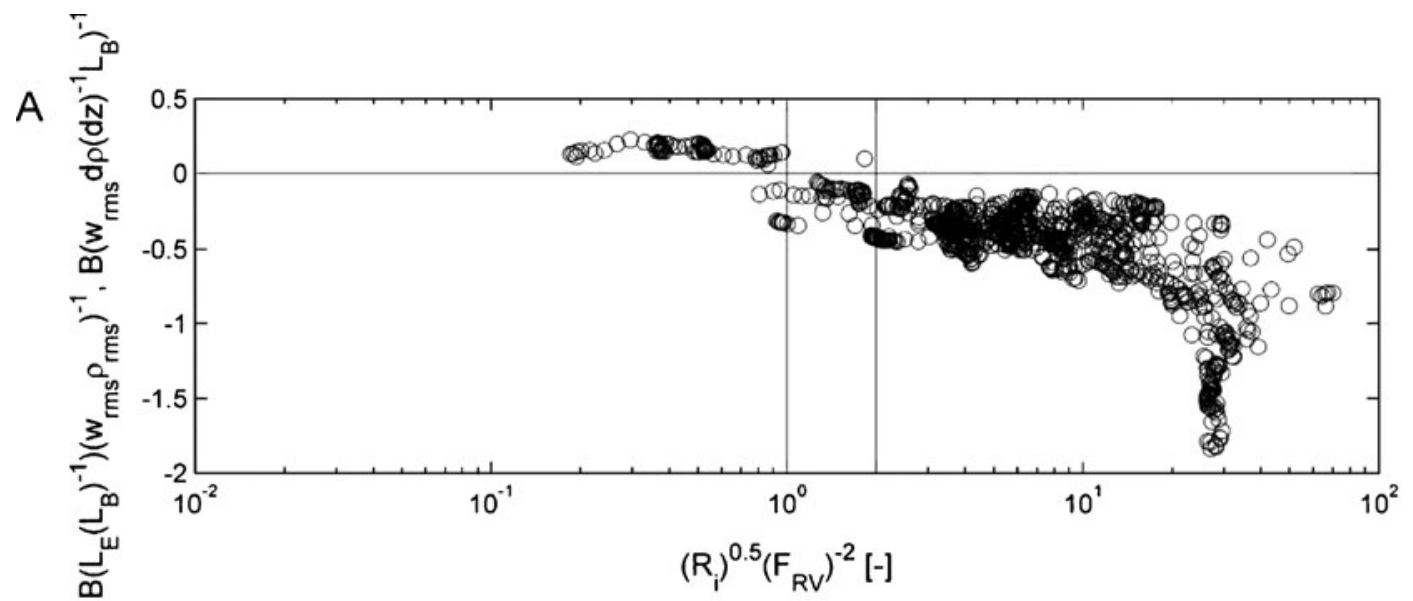

B

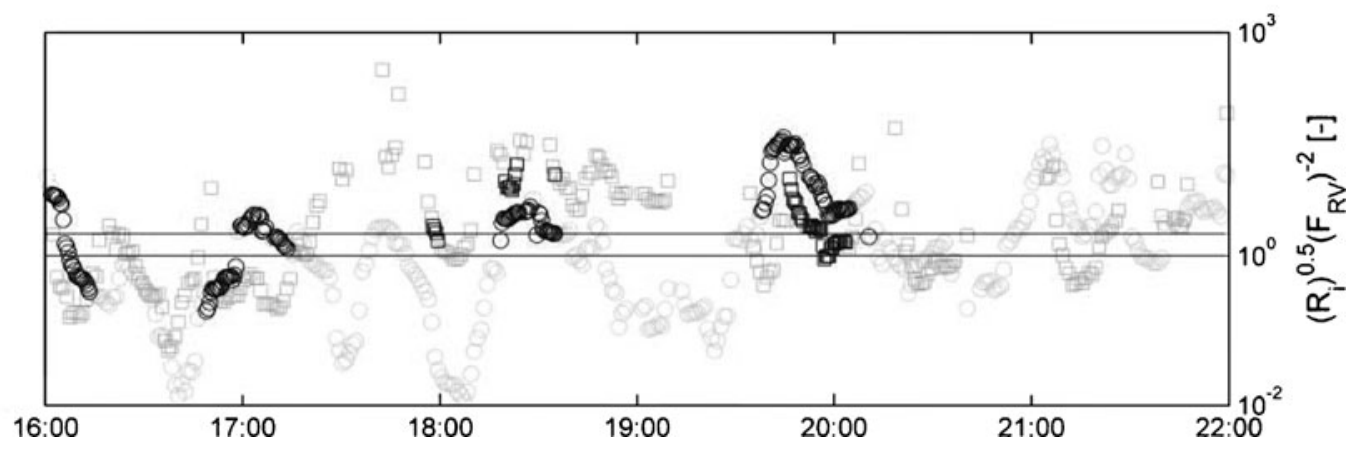

Fig. 15 Turbulence measurements recorded at the anchored station on April 16, 2006 (23 h of turbulence measurements). a Graph showing the dimensionless salinity-induced buoyancy flux vs. $\left((R i)^{1 / 2} \cdot\left(F r_{\mathrm{V}}\right)^{-2}\right)$. The vertical lines denote from left to right threshold values of 1 and 2 . b Graph showing time series of $\left((R i)^{1 / 2} \cdot\left(F r_{\mathrm{V}}\right)^{-2}\right)$ indicated by open circles and $\left((R i)^{1 / 2} \cdot\left(F r_{\mathrm{V}}\right)^{-2}\right)_{\mathrm{C}}$ indicated by open squares. The horizontal lines denote threshold values of 1 and 2. Data of disputable statistical significance are indicated by the gray color, while statistically significant data are depicted by the black color

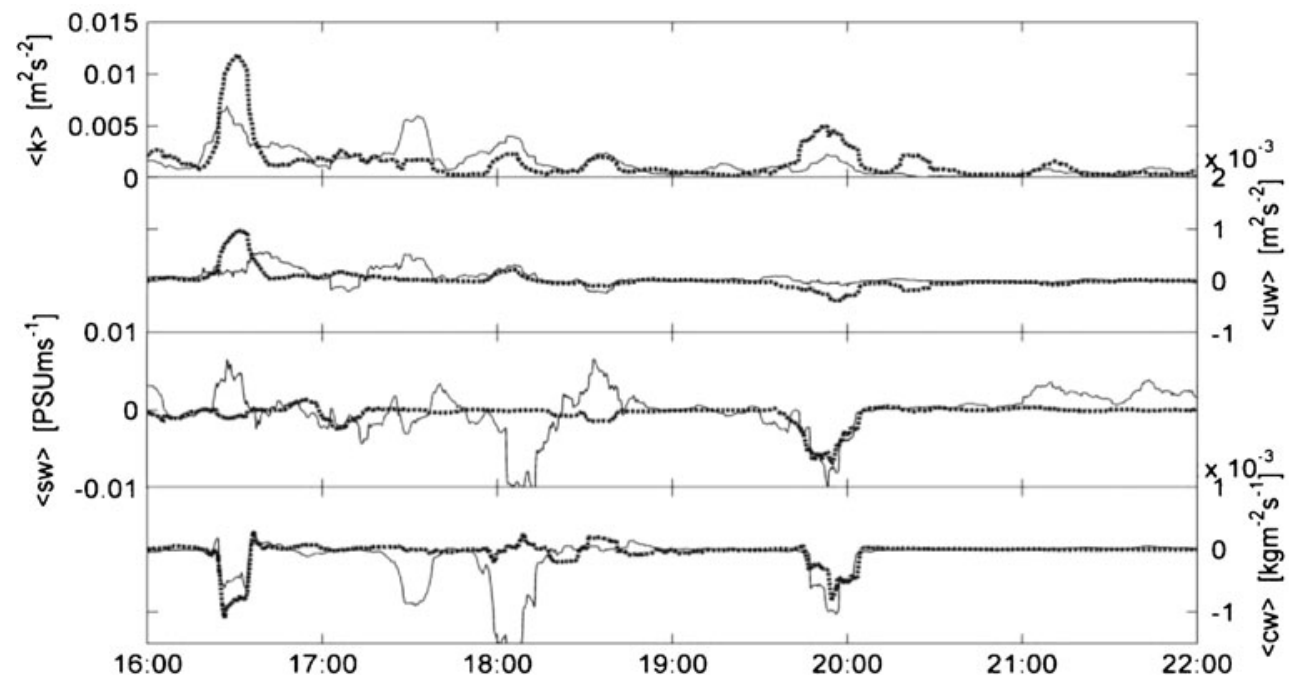

Fig. 16 Data recorded at the anchored station during the survey on April 16, 2006. From top to bottom: turbulent kinetic energy $(k=$ $\left.\left(<u^{2}>+<w^{2}>\right)\right)$, vertical turbulent transport of momentum $(<u w>)$, vertical turbulent transport of salinity $(\langle s w\rangle)$, and vertical turbulent transport of SPM $(\langle c w\rangle)$, respectively. Time (hours) is shown on the $x$-axis. Data at $0.30 \mathrm{~m}$ (dashed thick line) and $3.20 \mathrm{~m}$ (thin line) from the bed are shown. It is noted that the salinity stratification at $0.30 \mathrm{~m}$ was always stable. However, at $3.20 \mathrm{~m}$ between 1715 and 1815 hours, unstable salinity stratification events were observed. These events, associated with overturning motions, can explain the occurrence of negative buoyancy fluxes at that height 
Fig. 17 Turbulence measurements collected on April 16, 2006 recorded at the anchored station. a Graph showing < $\left(\gamma_{\mathrm{s}} s\right)^{2}>($ black $)$ and $<\gamma_{\mathrm{cs}} \mathrm{cs}>$ (gray). b Graph showing < $\left(\gamma_{\mathrm{c}} c\right)^{2}>($ black $)$ and $<\gamma_{\mathrm{cs}} \mathrm{cs}>$ (gray)
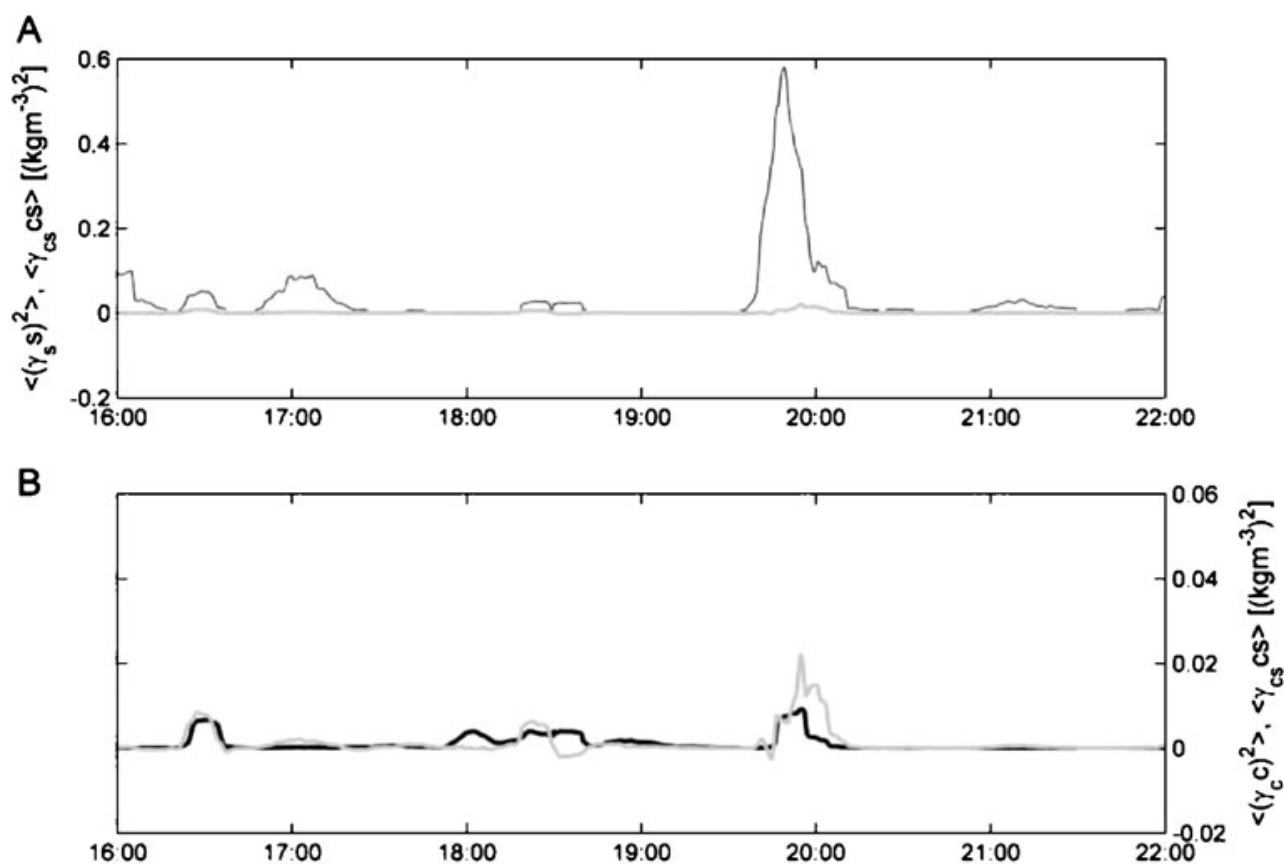

cant values for $\left((R i)^{1 / 2} \cdot\left(F r_{\mathrm{V}}\right)^{-2}\right)_{\mathrm{C}}$ below the threshold of 2 (Fig. 15b in Appendix 1, between 1800 and 1815 hours). Countergradient SPM buoyancy fluxes are observed at 1755 hours, between 1820 and 1830 hours, and between 1930 and 2000 hours (Fig. 16, lower graph, in Appendix 1). This is in agreement with values for $\left((R i)^{1 / 2}\right.$. $\left.\left(F r_{\mathrm{V}}\right)^{-2}\right)_{C}$ above the threshold of 2 at these periods (Fig. 15b, in Appendix 1). However, between 2000 and 2010 hours, SPM buoyancy fluxes are countergradient while $\left((R i)^{1 / 2} \cdot\left(F r_{\mathrm{V}}\right)^{-2}\right)_{C}$ values are below the threshold of 2 , and in most of the periods, countergradient SPM buoyancy fluxes correlate with countergradient salinity buoyancy fluxes. This indicates that salinity-induced buoyancy affects enhanced settling of SPM. Figure 17 in Appendix 1 (upper graph) shows that values for $<\left(\gamma_{s} s\right)^{2}>$ are generally larger than $\left\langle\gamma_{\mathrm{cs}} c s\right\rangle$, and the lower graph shows that values for $\left\langle\gamma_{\mathrm{cs}} c s\right\rangle$ are comparable (around 1630 hours) and larger than $<\left(\gamma_{c} c\right)^{2}>$ (around 1800 hours). This indicates that SPM descends to the bed mainly by salinity-induced buoyancy-driven motions, but SPMinduced buoyancy does play a role in the enhanced settling of SPM.
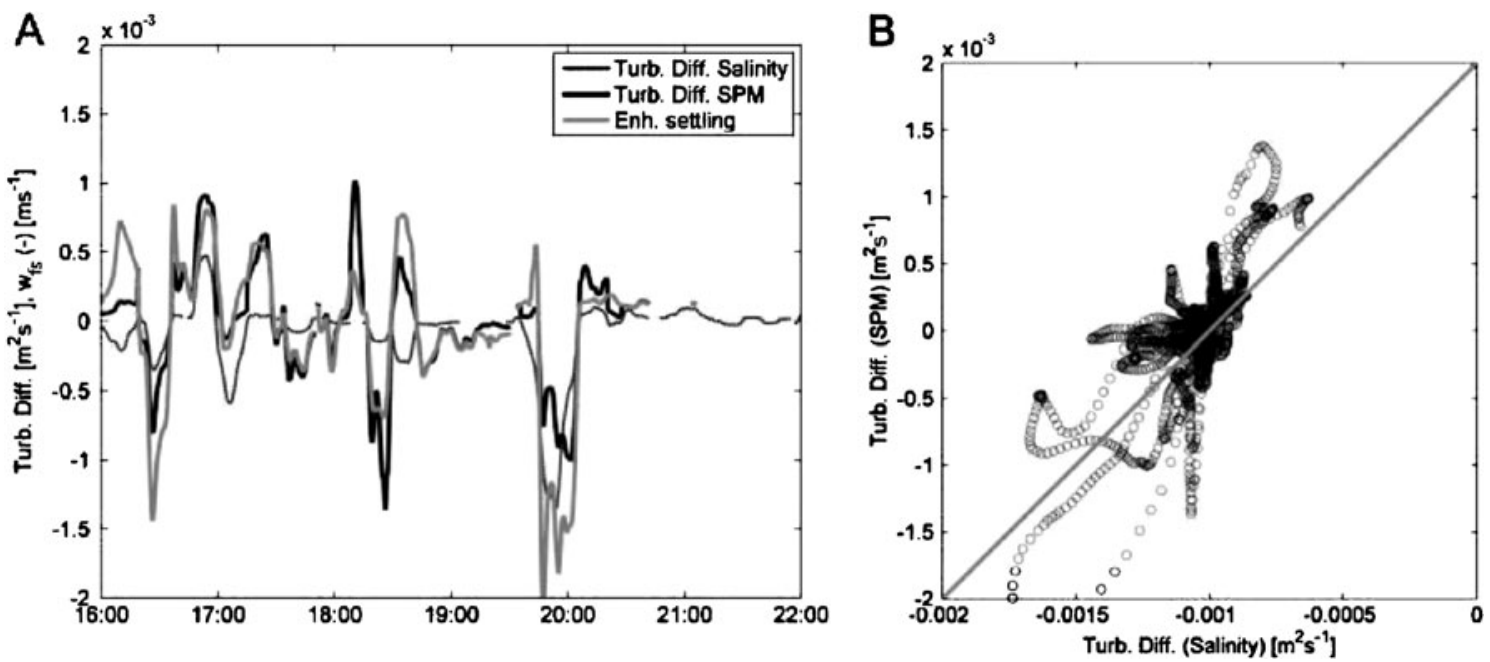

Fig. 18 Turbulence measurements collected on April 16, 2006 recorded at the anchored station. a Graph showing time series of estimates of turbulence diffusivities for salinity and SPM and estimates of fluid motion-induced settling velocities. b Graph showing a scatter plot of the turbulence diffusivity for salinity vs. the turbulence diffusivity for SPM. The solid line depicts perfect correlation 


\section{Appendix 2}

Estimates of turbulence diffusivities and fluid motion-induced settling velocities

In this appendix, we present time series of estimates of turbulence diffusivities for salinity and SPM and estimates of fluid motion-induced settling velocities (Fig. 18a, b in Appendix 2) for the measurements collected on April 16, 2006. The purpose was to establish the significance of the countergradient buoyancy fluxes in the vertical transport of mass.

Similar conclusions can be drawn from Fig. 18a, b in Appendix 2 as were already discussed for Fig. 7a, b. The magnitudes of the absolute value of the turbulence diffusivities for downgradient buoyancy fluxes and countergradient buoyancy fluxes are of the same order of magnitude. The values are generally orders of magnitude larger than the molecular diffusivity. Furthermore, the fluid motion-induced settling velocity (referred to as enhanced settling Fig. 18a in Appendix 2) is of the same order of magnitude as the gravitational settling velocity (MKO 1983; van Leussen 1994). Figure 18b Appendix 2 shows that the diffusivities for salinity and SPM do not show perfect correlation. This is expected based on the buoyancy correlation terms on the right-hand side of Eq. 8 (see discussion in Section 4.3).

\section{Appendix 3}

Memory effects induced by density fluctuations

In this appendix, the memory effects induced by the density fluctuations with increasing $R i$ are investigated for the measurements collected on April 16, 2006. Figure 19 in Appendix 3 shows a similar trend to Fig. 9. The binned ratio of APE to VKE increases with $R i$ until it reaches a maximum, after which the ratio decreases. This trend can be ascribed to differences in decay rates between APE and VKE, i.e., VKE decays generally faster than APE at lower $R i$ because positive buoyancy fluxes convert VKE to APE, while at larger $R i$, the decay rate of APE increases and the decay rate of VKE decreases due to the conversion of APE by countergradient buoyancy fluxes to VKE.

\section{Appendix 4}

Analysis of transports by intermittent motions

In this appendix, the transport by intermittent motions in the flow is investigated from data collected on April 16, 2006. Figure 20 in Appendix 4 shows that most of the covariance $(\langle s w\rangle,\langle c w\rangle)$ is contained at the low frequencies $\left(<0.1 \mathrm{~s}^{-1}\right)$. Ten-minute records at 1650 and 1955

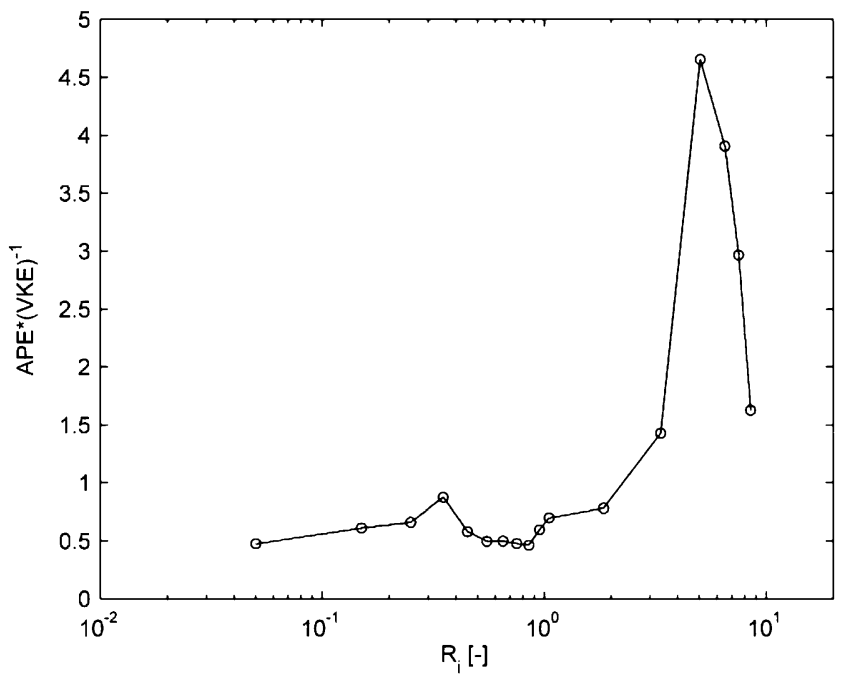

Fig. 19 Turbulence measurements collected at the anchored station on April 16, 2006. The graph shows binned values for APE(VKE) ${ }^{-1}$ vs. Ri. Note, however, that this ratio is not uniquely described by $R i$ 
Fig. 20 Co-spectral density (first panel of a, b) and absolute phase angles (second panel of $\mathbf{a}, \mathbf{b}$ ) of salinity (a) and SPM (b) fluctuations with vertical velocity fluctuations as a function of time (hours) and frequency calculated from data at $0.30 \mathrm{mab}$, collected at the anchored station on April 16, 2006. The black lines denote time instances (1650 hours and $R i<0.5,1955$ hours and $R i \approx 10$ ) where co-spectral density and absolute phase angles of $\langle u w\rangle$, $\langle s w\rangle$, and $\langle c w\rangle$ have been plotted as line graphs (Fig. 21)
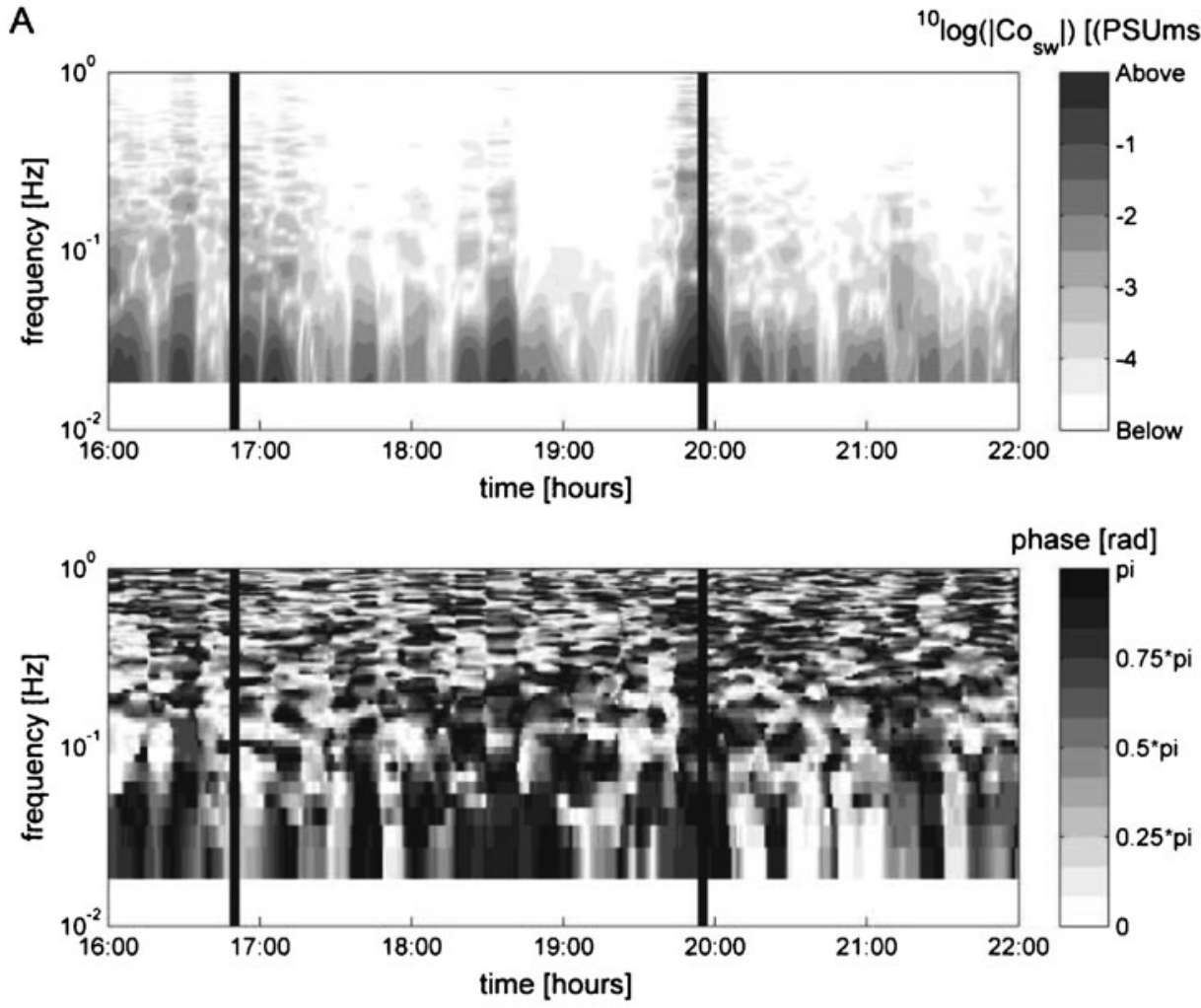

B
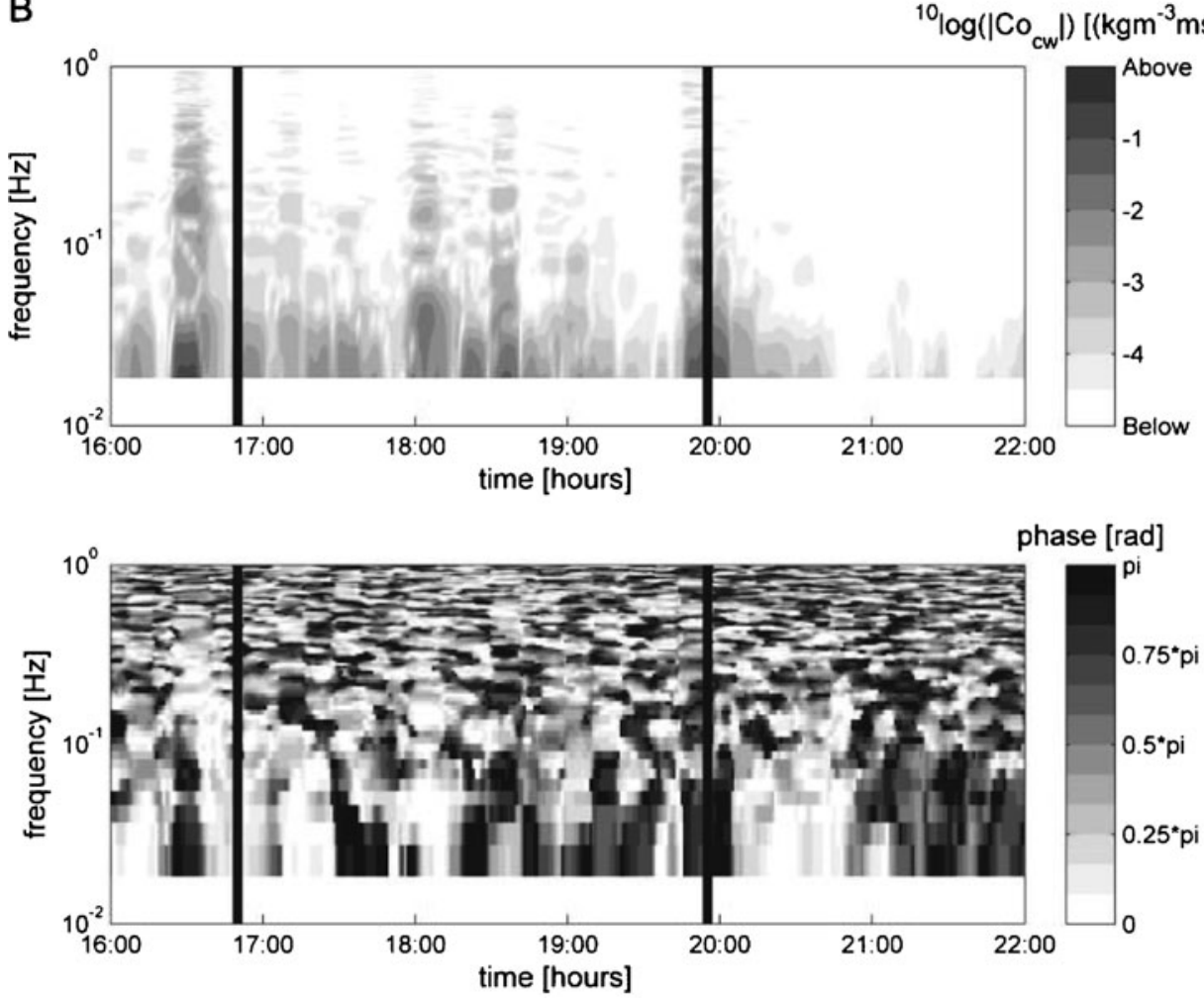
Fig. 21 a, b From top to bottom: normalized co-spectral density and absolute phase angles of $u$ and $w, s$ and $w$, and $c$ and $w$ as a function of $k\left(=\left(<U>\cdot f^{-1}\right)^{-1}\right)$ calculated from measurements collected on April 16, 2006 at $0.30 \mathrm{mab}$ at 1650 hours (a) $(R i<0.5)$ and at 1955 hours $(\mathbf{b})$ $(R i>5)$

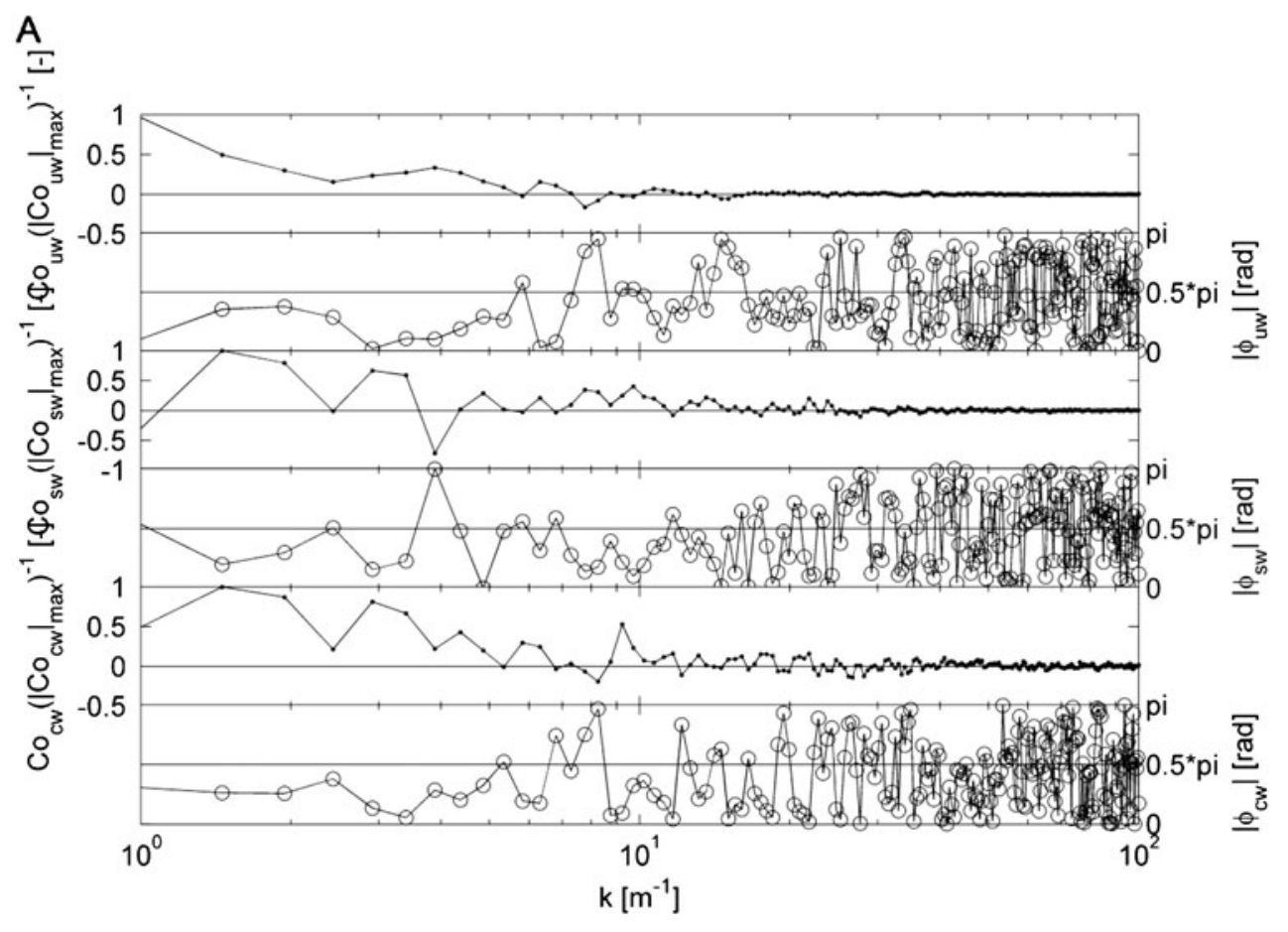

B

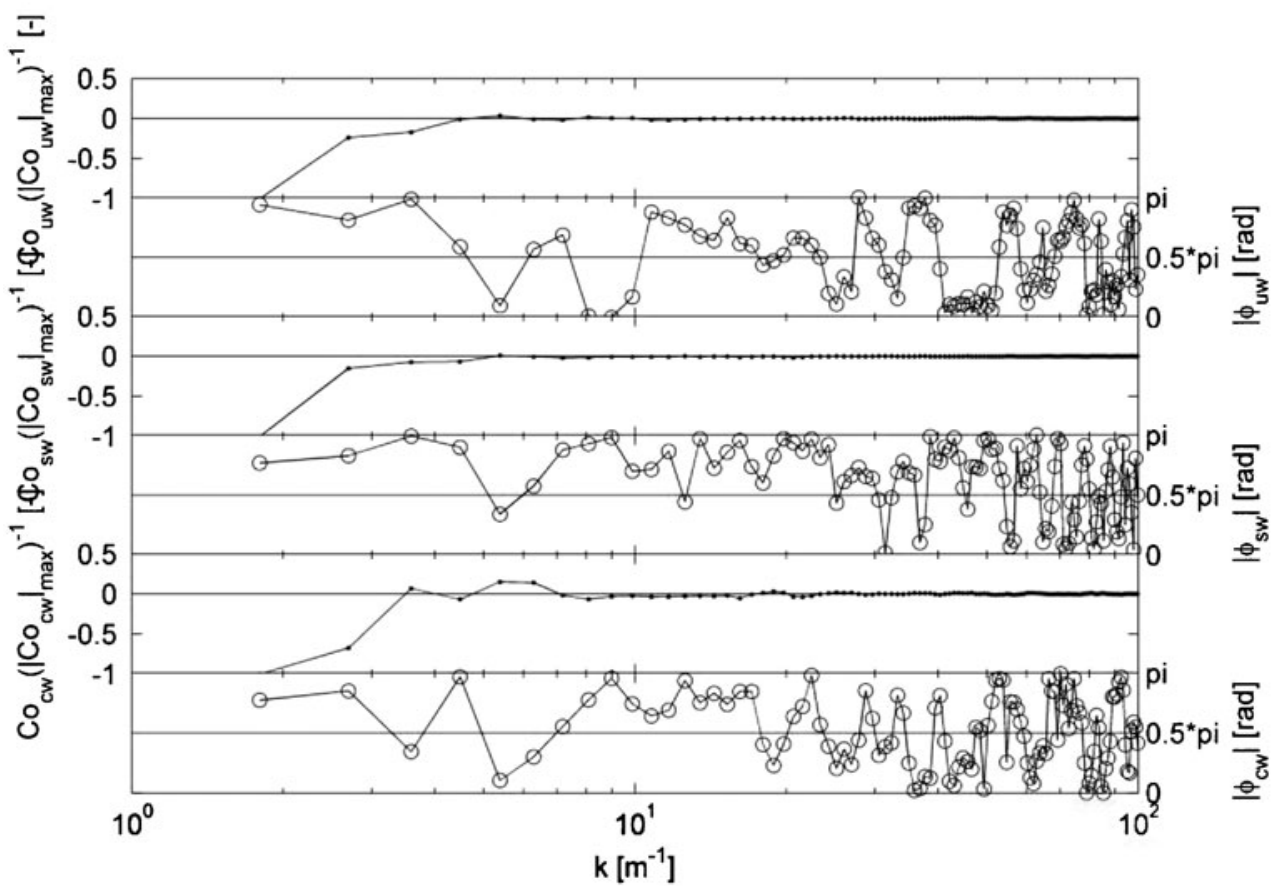

hours have been presented as line plots in Figs. 20 and 21 , respectively. Figures 19,20 and 21 show that at the low-frequency range $\left(<0.1 \mathrm{~s}^{-1}\right)$, around 1650 hours, $|\Delta \varphi|$ is between 0 and ${ }^{1 / 2} \pi$ (white and gray colors), indicative of mixing of salinity and SPM by energetic structures. Around 1955 hours, $|\Delta \varphi|$ is between ${ }^{1 / 2} \pi$ and $\pi$ (gray and black colors) in the range of negative buoyancy fluxes, which depicts countergradient transport.

Here, it is investigated how the contribution of different scales to the vertical momentum and buoyancy fluxes varies with $R i$. Figure 21a in Appendix 4 shows that $|\Delta \varphi|$ for $\langle u w\rangle,\langle s w\rangle$, and $\langle c w\rangle$ for wavenumbers $k<10$ are all 
within the range of active mixing, while the smaller scales exhibit small magnitude countergradient buoyancy fluxes. It can be seen from Fig. $21 \mathrm{~b}$ in Appendix 4 that with increasing $R i \gg>5$, the larger wavenumbers $k<10$ exhibit countergradient buoyancy fluxes as well. The expansion of countergradient fluxes from the smaller scale to the larger scale range with $R i$ is consistent with the conceptual model of Gerz and Schumann (1996). Furthermore, in most of the cases when $|\Delta \varphi| \approx^{1 / 2} \pi$ at wavenumbers $k<10$, the buoyancy fluxes are of small magnitude.

\section{References}

Adrian RJ, Meinhart CD, Tomkins CD (2000) Vortex organization in the outer region of the turbulent boundary layer. J Fluid Mech 422:1-54

Anwar HO (1983) Turbulence measurements in stratified and wellmixed estuarine flows. Estuar Coast Shelf Sci 17:243-260

Armenio V, Sarkar S (2002) An investigation of stably stratified turbulent channel flow using large-eddy simulation. J Fluid Mech 459:1-42

Burchard H, Petersen O, Rippeth TP (1998) Comparing the performance of the Mellor-Yamada and the $k-\varepsilon$ two-equation turbulence models. J Geophys Res 103:10543-10554

Davidson PA (2004) Turbulence: an introduction for scientists and engineers. Oxford University Press, Oxford, ISBN 9780198529484 , 0198529481

de Nijs MAJ, Winterwerp JC, Pietrzak JD (2009) On harbour siltation in the fresh-salt water mixing region. Cont Shelf Res 29(1):175193. doi:10.1016/j.csr.2008.01.019

de Nijs MAJ, Pietrzak JD, Winterwerp JC (2010a) Advection of the salt wedge and evolution of the internal flow structure in the Rotterdam Waterway. J Phys Oceanogr 2010:1-78. doi:10.1175/ 2010JPO4228.1

de Nijs MAJ, Winterwerp JC, Pietrzak JD (2010b) The effects of the internal flow structure on SPM entrapment in the Rotterdam Waterway. J Phys Oceanogr 40:2357-2380. doi:10.1175/ 2010JPO4233.1

Dyer KR, Christie MC, Manning AJ (2004) The effects of suspended sediment on turbulence within an estuarine turbidity maximum. Estuarine, Coast Shelf Sci 59:237-248

Etemad Shahidi SA, Imberger J (2006) Diapycnal mixing in the thermocline of lakes: estimations by different methods. Environ Fluid Mech 6:227-240

Gerz T, Schumann U (1996) A possible explanation of countergradient fluxes in homogeneous turbulence. Theor Comput Fluid Dyn 8:169-181

IEEE (1980) 1978 Practical salinity scale equations, Special issue on the practical salinity scale 1978. IEEE J Oceanic Eng OE-5(1):14

Itsweire EC, Helland KN, Van Atta CW (1986) The evolution of gridgenerated turbulence in a stably stratified fluid. J Fluid Mech 277:23-54

Ivey GN, Imberger J (1991) On the nature of turbulence in a stratified fluid. Part 1: the energetics of mixing. J Phys Oceanogr 21:650-658

Kawanisi K, Yokosi S (1993) Measurements of turbulence and suspended sediments in tidal river. J Hydraul Eng 119(6):704-724

Kay DJ, Jay DA (2003) Interfacial mixing in a highly stratified estuary 1. Characteristics of mixing. J Geophys Res 108:C3-C3072. doi:10.1029/2000JC000252
Komori S, Nagata K (1996) Effects of molecular diffusivities on counter-gradient scalar and momentum transfer in strongly stable stratification. J Fluid Mech 326:205-237

Komori S, Ueda H, Ogino F, Mizushina T (1983) Turbulence structure in stably stratified open-channel flow. J Fluid Mech 130:13-26

Launder BE (1975) On the effects of a gravitational field on the turbulent transport of heat and momentum. J Fluid Mech 67 (3):569-581

Lohrmann A, Hackett B, Røed LP (1990) High-resolution measurements of turbulence, velocity and stress using a pulse-to-pulse coherent sonar. J Atmos Oceanic Technol 7:19-37

Lu SS, Willmarth WW (1973) Measurements of the structure of the Reynolds stress in a turbulent boundary layer. J Fluid Mech 60 (3):481-511

Mellor GL, Yamada T (1974) A hierarchy of turbulence closure models for planetary boundary layers. J Atmos Sci 31:1791-1806

Miles JW (1976) On the stability of heterogeneous shear flows. J Fluid Mech 10:496-508

MKO (1983) Minimalisering Kosten Onderhoudsbaggerwerk, Een bundeling van kennis en ervaring in de strijd tegen het slib (in Dutch)

Monin AS, Obukhov AM (1953) Dimensionless characteristics of turbulence in the surface layer of the atmosphere. Dokl Akad Nauk SSSR 93(2):223-226 (in Russian)

Munk WH, Anderson ER (1948) Notes on the theory of the thermocline. J Mar Res 7:276-295

Natrajan VK, Christensen KT (2006) The role of coherent structures in subgrid-scale energy transfer within the log layer of wall turbulence. Phys Fluids 18:1-13

Nezu I, Nakagawa H (1993) Turbulence in open-channel flows. International Association for Hydraulic Research, A.A. Balkema, Rotterdam/Brookefield

Nikora VI, Goring DG (2002) Fluctuations of suspended sediment concentration and turbulent sediment fluxes in an open-channel flow. J Hydraul Eng 128(2):214-224

Nunes Vaz RA, Simpson JH (1994) Turbulence closure modeling of estuarine stratification. J Geophys Res 99(C8):1614316160

Osborn TR (1980) Estimates of the local rate of vertical diffusion from dissipation measurements. J Phys Oceanogr 10:83-89

Osborn TR, Cox CS (1972) Oceanic fine structure. Geophys Fluid Dyn 3:321-345

Ozmidov RV (1965) On the turbulence exchange in stably stratified ocean. Izv Atmos Ocean Phys Ser 1(8):853-860

Peters H (1997) Observations of stratified turbulent mixing in an estuary: neap-to-spring variations during high river flow. Estuar Coast Shelf Sci 45:69-88

Riley JJ, Lelong MP (2000) Fluid motions in the presence of strong stable stratification. Annu Rev Fluid Mech 32:613-657

Rippeth TP, Simpson JH, Williams E, Inall ME (2003) Measurement of the rates of production and dissipation of turbulent kinetic energy in an energetic tidal flow: red wharf bay revisited. J Phys Oceanogr 33:1889-1901

Rohr JJ, Itsweire EC, Helland KN, van Atta CW (1988) Growth and decay of turbulence in a stably stratified shear flow. J Fluid Mech 195:77-111

Schumann U (1987) The countergradient heat flux in turbulent stratified flows. Nucl Eng Des 100:255-262

Smyth WD, Moun JN (2000) Length scales of turbulence in stably stratified mixing layers. Phys Fluids 12:1327-1341

Soulsby RL (1980) Selecting record length and digitization rate for near-bed turbulence measurements, J Phys Oceanogr 10:208219 
Stacey MT, Monismith SG, Burau JR (1999) Measurements of Reynolds stress profiles in tidal flows. J Geophys Res 104 (C5):10933-10949

Taylor JR, Sarkar S, Armenio V (2005) Large eddy simulation of stably stratified open channel flow, 2005. Phys Fluids 17:116602$1-116602-17$

Tropea C (1983) A note concerning the use of a one-component LDA to measure shear stress terms. Exp Fluids 1(10):209-210

Turner JS (1973) Buoyancy effects in fluids, Cambridge University Press, ISBN: $052108623 \mathrm{x}, 367 \mathrm{pp}$

van der Ham R (1999) Turbulent exchange of fine sediments in a tidal flow. Commun Hydraul Geotech Eng. ISSN 0169-6548, Report No. $99-1$

van Leussen W (1994) Estuarine macroflocs and their role in fine-grained sediment transport. $\mathrm{PhD}$ thesis, Utrecht University, p. 484
Venayagamoorthy SK, Strecth DD (2006) Lagrangian mixing in decaying stably stratified turbulence. J Fluid Mech, 564, pp. 197226

West JR, Knight DW, Shiono K (1986) Turbulence measurements in the Great Ouse estuary, J Hydraul Eng, 112(3):167-180

West JR, Oduyemi KOK (1989) Turbulence measurements of suspended solids concentration in estuaries. J Hydraul Eng 115 (4):457-474

West JR, Shiono K (1988) Vertical mixing processes on ebb tides in a partially mixed estuary. Estuar Coast Shelf Sci 26:5166

Zdravkovich MM (1997) Flow around circular cylinders: a comprehensive guide through flow phenomena, experiments, applications, mathematical models, and computer simulations. Oxford University Press, Oxford, p 694 